\title{
Edge-swapping algorithms for the minimum fundamental cycle basis problem
}

\author{
Edoardo Amaldi \\ DEI, Politecnico di Milano, Piazza L. da Vinci 32, 20133 Milano, Italy \\ Leo Liberti \\ LIX, École Polytechnique, F-91128 Palaiseau, France \\ Francesco Maffioli \\ DEI, Politecnico di Milano, Piazza L. da Vinci 32, 20133 Milano, Italy \\ Nelson Maculan \\ COPPE, Universidade Federal do Rio de Janeiro, P.O. Box 68511, 21941-972, \\ Rio de Janeiro, Brazil \\ Submitted: 30 September 2006
}

\begin{abstract}
We consider the problem of finding a fundamental cycle basis with minimum total cost in an undirected graph. This problem is NP-hard and has several interesting applications. Since fundamental cycle bases correspond to spanning trees, we propose a local search algorithm, a tabu search and variable neighborhood search in which edge swaps are iteratively applied to a current spanning tree. We also present a mixed integer programming formulation of the problem whose linear relaxation yields tighter lower bounds than other known formulations. Computational results obtained with our algorithms are compared with those from the best available constructive heuristic on several types of graphs. ${ }^{1}$
\end{abstract}

Keywords: cycle basis, fundamental cycle, fundamental cut, edge swap, heuristic, metaheuristic.

$\overline{1}$ This article extends the conference paper [1].

Email addresses: amaldi@elet.polimi.it (Edoardo Amaldi),

liberti@lix.polytechnique.fr (Leo Liberti), maffioli@elet.polimi.it

(Francesco Maffioli), maculan@cos.ufrj.br (Nelson Maculan). 


\section{Introduction}

Let $G=(V, E)$ be a simple undirected graph with $n$ nodes and $m$ edges, weighted by a non-negative cost function $w: E \rightarrow \mathbb{R}^{+}$; we use the notation $w(F)=\sum_{e \in F} w_{e}$ for subsets $F \subseteq E$. A cycle is a subset $\gamma$ of $E$ such that every node of $V$ is incident with an even number of edges in $\gamma$. Since an elementary cycle is a connected cycle such that at most two edges are incident to any node, cycles can be viewed as the (possibly empty) union of edge-disjoint elementary cycles. If cycles are considered as edge-incidence binary vectors in $\{0,1\}^{|E|}$, it is well-known that the cycles of a graph form a vector space over $G F(2)$. When the graph $G$ is connected, a set of $\nu=m-n+1$ cycles is a cycle basis if it is a basis in this cycle vector space associated to $G$. There are special cycle bases that can be derived from the spanning trees of $G$. Let $T \subseteq E$ denote the edge set of any spanning tree of $G$; the edges in $T$ are called branches of the tree, and those in $E \backslash T$ (the co-tree) are called the chords of $G$ with respect to $T$ (or, with a slight abuse of notation, the chords of $T$ ). Any chord uniquely identifies a cycle consisting of the chord itself and the unique path in $T$ connecting the two nodes of the chord. These $\nu$ cycles are called fundamental cycles and they form a Fundamental Cycle Basis (FCB) of $G$ with respect to $T$ (see below for the definition of weakly FCBs). Since the cycle space of a graph is the direct sum of the cycle spaces of its edge-biconnected components, we assume that $G$ is edge-biconnected, i.e., $G$ contains at least two edge-disjoint paths between any pair of nodes.

Figure 1 shows the difference between a non-fundamental cycle basis and a fundamental one.
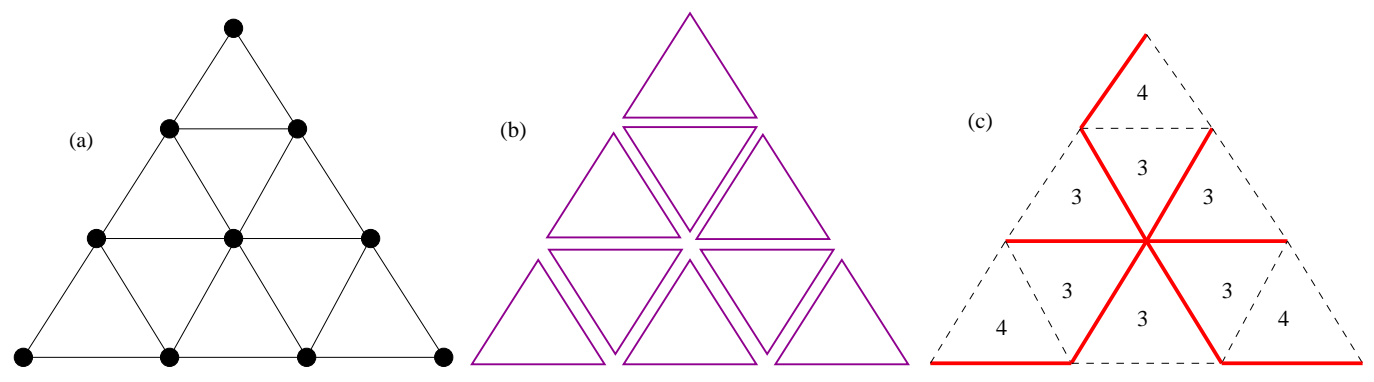

Fig. 1. (a) A triangular grid graph with unit edge costs, (b) a minimum cycle basis with cost 27 and (c) a minimum FCB with cost 30 .

In this paper we consider the following problem.

Min FCB: Given an edge-biconnected graph $G=(V, E)$ with non-negative costs assigned to the edges, find a fundamental cycle basis $\left\{\gamma_{1}, \ldots, \gamma_{\nu}\right\}$ of minimum total cost, i.e., which minimizes $\sum_{i=1}^{\nu} w\left(\gamma_{i}\right)$ where $w\left(\gamma_{i}\right)$ denotes the sum of the costs of all edges in cycle $\gamma_{i}$. 
Previous and related work. Cycle bases have been used in the field of electrical networks since the time of Kirchoff [2]. Interest in minimum FCBs arises in a variety of application fields, such as electrical circuit testing [3], generating minimal perfect hash functions (used in compiler design) [4], planning cyclic timetables [5,6], coding of ring compounds [7] and planning complex syntheses in organic chemistry [8]. Many of the early results about the graph-theoretical structure of FCBs are due to a sequence of papers by Sysło [9-14]. In particular, a cycle basis is fundamental if and only if each cycle in the basis contains at least one edge which is not contained in any other cycle in the basis [12]. Moreover, two spanning trees whose symmetric difference is a collection of 2-paths (paths where each node, excluding the endpoints, has degree 2) give rise to the same FCB. An algorithm for enumerating the FCBs is also presented in [14]. More recently, weakly fundamental cycle bases, in which it suffices that there exists an ordering of the cycles $\gamma_{1}, \ldots, \gamma_{\nu}$ such that $\gamma_{j} \backslash\left(\gamma_{1} \cup \ldots \cup \gamma_{j-1}\right) \neq \emptyset$ for all $j, 2 \leq j \leq \nu$, have also been considered, see for instance [15]. Notice that for a cycle basis to be fundamental it has to satisfy the above condition for all possible orderings of the cycles. In this work we focus on fundamental cycle bases that are fundamental and, for the sake of brevity, do not mention strictness.

Although the problem of finding a general cycle basis of minimum total cost can be solved in polynomial time (see [16] and the recent improvements $[17,18]$ ), requiring (strict) fundamentality makes the problem NP-hard [19]. The approximability of MIN FCB is addressed in [20], where the problem is shown to be APX-hard even when restricted to unweighted graphs, a $O\left(\log ^{2} n \log \log n\right)$-approximation algorithm is given for arbitrary graphs, and tighter approximability bounds are obtained for dense graphs, including a polynomial-time approximation scheme for complete graphs. Constructive heuristic methods for solving the Min FCB problem have been proposed in $[4,19,21]$, and tested on a variety of regular and randomly generated instances.

A well-known problem related to MiN FCB is the minimum routing cost spanning tree problem, see e.g. [22,23]. Given a weighted graph $G$ as in Min FCB, one looks for a spanning tree $T$ of $G$ which minimizes the sum of the costs of the paths on $T$ between all pairs of nodes. In spite of the similarities, Min FCB differs substantially from this problem since the sum is taken only over the pairs of nodes corresponding to the chords of $T$. Note that for weighted graphs, the cost of all chords is also included in the objective function.

The paper is organized as follows. In Section 2 we describe a local search algorithm in which the spanning tree associated to the current FCB is iteratively modified by performing edge swaps. In Section 3 the same type of edge swaps is adopted within two metaheuristic schemes, namely a variable neighborhood search and a tabu search. To provide lower bounds on the cost of optimal solutions, a new mixed integer programming (MIP) formulation of the problem 
is presented in Section 4. Computational results are reported and discussed in Section 5 .

\section{Edge-swapping local search}

Due to the correspondence between fundamental cycle bases and spanning trees and the fact that the set of all spanning trees can be efficiently explored by iteratively swapping pairs of edges (see e.g. [24,25]), we consider a local search algorithm for Min FCB based on edge swaps. Starting from the spanning tree associated to an initial $\mathrm{FCB}$, at each iteration we swap a chord $e$ with one of the branches in the fundamental cycle induced by $e$ so that the total FCB cost is decreased, until the cost cannot be further decreased, i.e., a local minimum is found. In practice, it was verified experimentally that allowing swaps that keep the objective function value constant results in a more effective exploration of the solution space.

For each chord $e=\{u, v\}$ of $G$ with respect to the spanning tree $T$, let $\langle u, v\rangle$ be the unique path connecting $u, v$ in $T$ and let $\gamma_{T}^{e}=\langle u, v\rangle \cup\{u, v\}$ the unique fundamental cycle induced by $e$. For each branch $b$ of $T$, the removal of $b$ from $T$ induces the partition of the node set $V$ into two subsets $S_{T}^{b}$ and $\bar{S}_{T}^{b}$. Denote by $\delta_{T}^{b}$ the fundamental cut of $G$ induced by the branch $b$ of $T$, i.e., $\delta_{T}^{b}=\delta\left(S_{T}^{b}\right)=\left\{\{u, v\} \in E \mid u \in S_{T}^{b}, v \in \bar{S}_{T}^{b}\right\}$. It is easy to verify that each chord $e$ belongs to all fundamental cuts $\delta_{T}^{b}$ induced by the branches $b$ of the fundamental cycle $\gamma_{T}^{e}$. Denoting by $\mathcal{C}(T)$ the set of cycles in the FCB associated to $T$, then $w(\mathcal{C}(T))$ denotes the cost of this FCB.

\subsection{Initial solutions}

Initial solutions are obtained by applying a very fast "tree-growing" procedure [21], where a spanning tree and its corresponding FCB are generated by adding nodes to the tree according to predefined criteria. The adaptation of Paton's procedure to the Min FCB problem proceeds as follows. Initially the node set $V_{T}$ of the tree only contains a root node $v_{0}$, and the set $W$ of nodes to be examined is taken as $V$. Then at each step a node $u \in W \cap V_{T}$ is selected according to a predefined ordering. For all nodes $z$ adjacent to $u$, if $z \notin V_{T}$, the edge $\{z, u\}$ is included in $T$ (the edge is selected), the node $z$ is added to $V_{T}$ and the node $u$ is removed from $W$. Nodes to be examined are selected according to non-increasing degree and, to break ties, to increasing edge star costs. The resulting order tends to maximize the chances of identifying short fundamental cycles early in the process. The performance of this procedure is comparable to other existing tree-growing techniques $[19,4]$. Ini- 
tial solutions can also be obtained via the approximation algorithm given in [26] for constructing spanning trees with average stretch $O\left((\log n)^{2} \log \log n\right)$ in time $O\left(m \log n+n(\log n)^{2}\right)$. The average stretch of a tree $T$ is defined as $\operatorname{avestretch}(T)=\frac{1}{|E|} \sum_{\{u, v\} \in E} \frac{w_{T}(u, v)}{w_{u v}}$, where $w_{T}(u, v)=\sum_{\{x, y\} \in\langle u, v\rangle} w_{x y}$. The relation between average stretch and FCB cost is given by

$$
\begin{aligned}
|E| \operatorname{avestretch}(T) & =\sum_{\{u, v\} \in E} \frac{w_{T}(u, v)}{w_{u v}}=\sum_{b \in T} 1+\sum_{\{u, v\} \in E \backslash T} \frac{w_{T}(u, v)}{w_{u v}} \\
& =|T|+\sum_{e \in E \backslash T} \frac{w\left(\gamma_{T}^{e}\right)-w_{e}}{w_{e}} \\
& =|T|+\sum_{e \in E \backslash T} \frac{w\left(\gamma_{T}^{e}\right)}{w_{e}}-(|E|-|T|) \\
& =\sum_{e \in E \backslash T} \frac{w\left(\gamma_{T}^{e}\right)}{w_{e}}+2 n-m-2,
\end{aligned}
$$

that is an FCB-like cost where the cost of each cycle is scaled by the weight of the corresponding chord, plus an additive constant. For unweighted graphs where $w_{e}=1$ for all $e \in E$, this becomes simply $w(\mathcal{C}(T))+2 n-m-2$ and the approximation guarantee for the average stretch then translates directly into an approximation guarantee for the FCB. For the weighted graphs, a related technique yielding an approximation of $O\left(w(E) \log n \log ^{2} n\right)$ is given in [27].

\subsection{Selection of a best edge swap}

Let $\pi=(e, b)$, where $e$ is a chord and $b$ is a branch of $\gamma_{T}^{e}$, be the edge swap such that:

$$
\pi T=T \cup\{e\} \backslash\{b\} .
$$

An edge swap $\pi=(e, b)$, with $b \in \gamma_{T}^{e}$, is an improving edge-swap for $T$ if it decreases the total FCB cost, that is if $\Delta_{\pi}=w(\mathcal{C}(T))-w(\mathcal{C}(\pi T))>0$.

In our edge-swapping local search algorithm, we start from the FCB associated to the spanning tree provided by the constructive procedure of Section 2.1. Then at each iteration we apply to the current spanning tree $T$ the edge swap $\pi=(e, b)$ which yields a maximum decrease in the objective function. The algorithm terminates when no improving edge swap exists for the current spanning tree.

At each iteration of the edge-swapping local search, we consider the spanning tree $T$ of $G$ associated to the current FCB. Two procedures are needed: $\mathrm{FCBCOST}(T)$, a procedure which computes the cost of the fundamental cycle basis associated with $T$, and a procedure $\operatorname{BESTSWhaP}(T)$, which finds a chord 


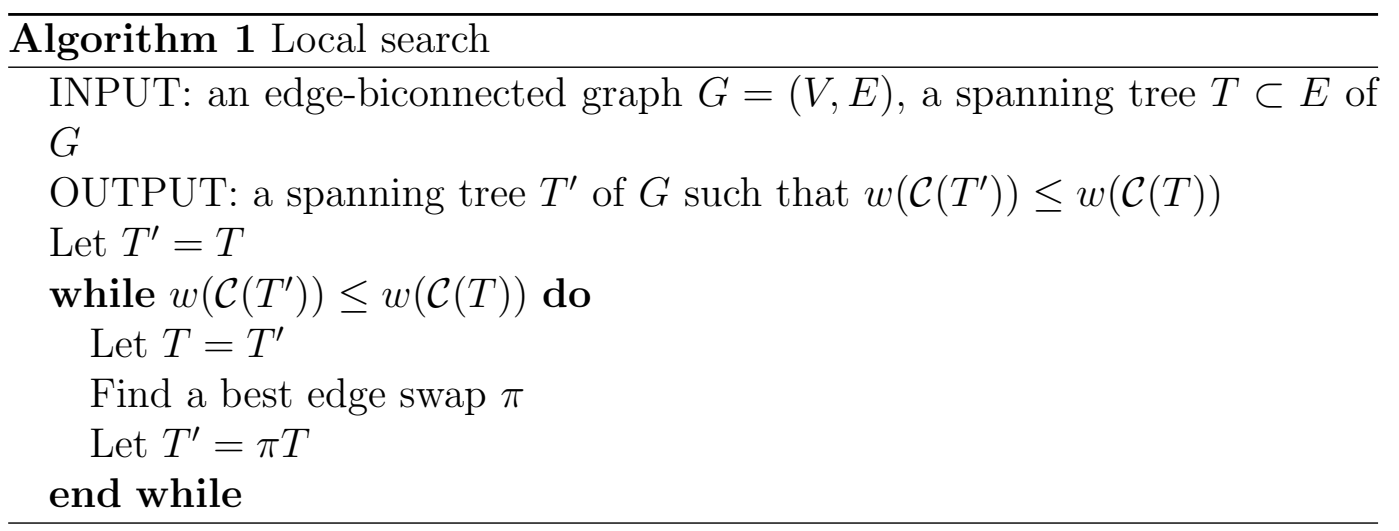

and a branch of the induced fundamental cycle whose swap yields the largest decrease in the objective function. We show next that an edge swap $\pi=(e, b)$ where $e$ is a chord and $b$ a branch of $\gamma_{T}^{e}$, acts on a fundamental cycle $\gamma_{T}^{f}$, where $f$ is another chord of $T$, by either fixing it (i.e. by leaving it unchanged), or by mapping it to the symmetric difference of $\gamma_{T}^{f}$ and $\gamma_{T}^{e}$.

The following elementary facts are stated without proof.

(1) For any edge swap $\pi=(e, b)$, where $e$ is a chord and $b$ a branch of $\gamma_{T}^{e}$, $\pi$ fixes $\gamma_{T}^{e}$, and, if $f \neq e$ is another chord of $T$ such that $\gamma_{T}^{e} \cap \gamma_{T}^{f}=\emptyset, \pi$ also fixes $\gamma_{T}^{f}$.

(2) For any pair of chords $e, f$ of $T$, there exists a branch $b$ of $T$ such that $e, f \in \delta_{T}^{b}$ if and only if $b \in \gamma_{T}^{e} \cap \gamma_{T}^{f}$.

By Fact (1), the only case where a swap $\pi=(e, b)$ does not fix a fundamental cycle $\gamma^{f}$, for a different chord $f$ of $T$, is when $\gamma_{T}^{e} \cap \gamma_{T}^{f} \neq \emptyset$. Figure 2 illustrates the following result.

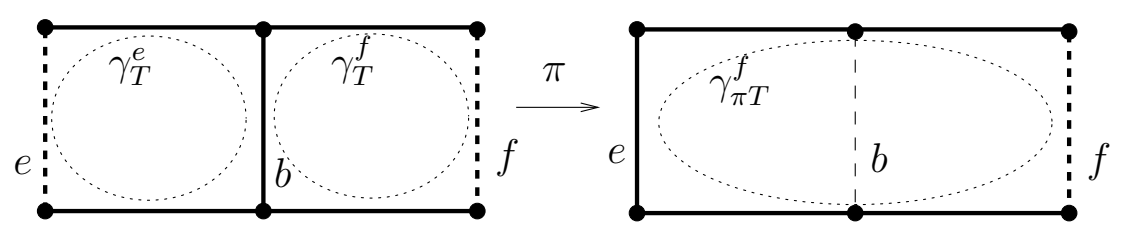

Fig. 2. Illustration of the statement of Theorem 1.

Theorem 1 Consider an edge swap $\pi=(e, b)$, where $e$ is a chord and $b$ a branch of $\gamma_{T}^{e}$. For any chord $f \neq e$ of $T$, if $b \in \gamma_{T}^{e} \cap \gamma_{T}^{f}$, then once the swap $\pi$ is performed the fundamental cycle induced by the chord $f$, denoted by $\gamma_{\pi T}^{f}$ or $\pi\left(\gamma_{T}^{f}\right)$, is such that $\gamma_{\pi T}^{f}=\gamma_{T}^{f} \triangle \gamma_{T}^{e}$.

Proof. First, by Fact (2) we have that $e, f \in \delta_{T}^{b}$. Then we need the two following claims, which are illustrated in Figure 3.

Claim 1. For all $h \in \delta_{T}^{b}$ such that $h \neq b, \gamma_{T}^{h} \cap \delta_{T}^{b}=\{b, h\}$.

Proof. Since $\gamma_{T}^{h}$ is the simple cycle consisting of $h$ and the unique path in $T$ connecting the endpoints of $h$ through $b$, the only edges that belong both to 
the cycle and to the cut of $b$ are $b$ and $h$.

Claim 2. For all pairs of chords $g, h \in \delta_{T}^{b}$ such that $g \neq h$ there exists a unique simple cycle $\gamma \subseteq G$ such that $g \in \gamma, h \in \gamma$, and $\gamma \backslash\{g, h\} \subseteq T$.

Proof. Let $g=\left\{g_{1}, g_{2}\right\}, h=\left\{h_{1}, h_{2}\right\}$ and assume w.l.o.g. that $g_{1}, h_{1}, g_{2}, h_{2}$ are labeled so that $g_{1}, h_{1} \in S_{T}^{b}$ and $g_{2}, h_{2} \in \bar{S}_{T}^{b}$. Since there exist unique paths $p \subseteq T$ connecting $g_{1}, h_{1}$ and $q \subseteq T$ connecting $g_{2}, h_{2}$, the edge subset $\gamma=\{g, h\} \cup p \cup q$ is a cycle with the required properties. Assume now that there is another cycle $\gamma^{\prime}$ with the required properties. Then $\gamma^{\prime}$ defines paths $p^{\prime}, q^{\prime}$ connecting respectively $g_{1}, h_{1}$ and $g_{2}, h_{2}$ in $T$. Since $T$ is a spanning tree, $p=p^{\prime}$ and $q=q^{\prime}$, and hence $\gamma^{\prime}=\gamma$.

Consider the cycle $\gamma=\gamma_{T}^{e} \triangle \gamma_{T}^{f}$. By hypothesis, $b \in \gamma_{T}^{e} \cap \gamma_{T}^{f}, e \in \gamma_{T}^{e}, f \in$ $\gamma_{T}^{f}$. Since $e, f \in \delta_{T}^{b}$, by Claim $1 f \notin \gamma_{T}^{e}$ and $e \notin \gamma_{T}^{f}$. Thus $e, f \in \gamma$ and $b \notin \gamma$. Consider now $\pi\left(\gamma_{T}^{f}\right)$ : since $b \in \gamma_{T}^{f}$ and $\pi=(e, b)$, we have $e \in \pi\left(\gamma_{T}^{f}\right)$. Furthermore, since $\pi$ fixes $f, f \in \pi\left(\gamma_{T}^{f}\right)$. Hence, by Claim 2, we have that $\pi\left(\gamma_{T}^{f}\right)=\gamma=\gamma_{T}^{f} \triangle \gamma_{T}^{e}$.

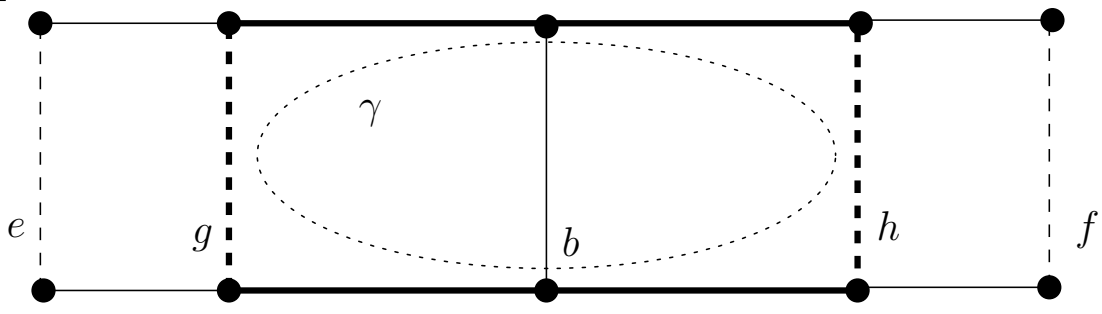

Fig. 3. Illustration of Claims 1 and 2 in the proof of Theorem 1.

From Theorem 1, it follows that the change $\Delta_{\pi}$ in FCB cost due to an edge swap $\pi=(e, b)$, where $e$ is a chord and $b$ a branch of the current spanning tree $T$ belonging to $\gamma_{T}^{e}$, only depends on the change in cost of those fundamental cycles $\gamma_{T}^{f}$ (where $f \neq e$ is a chord of $T$ ) which contain $b$.

Corollary 2 Let $\pi=(e, b)$ be an edge swap with $e$ a chord and $b$ a branch of $\gamma_{T}^{e}$, and let $F(b)=\left\{f \in E \backslash T \mid b \in \gamma_{T}^{f}\right\}$. Then

$$
\Delta_{\pi}=2 \sum_{f \in F(b)} w\left(\gamma_{T}^{e} \cap \gamma_{T}^{f}\right)-|F(b)| w\left(\gamma_{T}^{e}\right)
$$

Proof. By Theorem 1, the only fundamental cycles that change under the action of $\pi$ are those that contain $b$, and can therefore be used to quantify the change $\Delta_{\pi}$. We have: 


$$
\begin{aligned}
\Delta_{\pi} & =\sum_{f \in F(b)}\left(w\left(\gamma_{T}^{f}\right)-w\left(\gamma_{\pi T}^{f}\right)\right)= \\
& =\sum_{f \in F(b)}\left(w\left(\gamma_{T}^{f}\right)-w\left(\gamma_{T}^{e} \triangle \gamma_{T}^{f}\right)\right)= \\
& =\sum_{f \in F(b)}\left(2 w\left(\gamma_{T}^{e} \cap \gamma_{T}^{f}\right)-w\left(\gamma_{T}^{e}\right)\right)
\end{aligned}
$$

because $w\left(\gamma_{T}^{e} \triangle \gamma_{T}^{f}\right)=w\left(\gamma_{T}^{e}\right)+w\left(\gamma_{T}^{f}\right)-2 w\left(\gamma_{T}^{e} \cap \gamma_{T}^{f}\right)$. The result follows.

In the rest of this section, we present two different techniques to determine a best edge swap at each iteration. The first one, described in Section 2.3, uses least common ancestors to identify fundamental cycles. The second one, described in Section 2.4, efficiently updates lists of fundamental cuts and cycles by using symmetric differences of edge sets. As we shall see in Section 2.5, although the worst-case complexity estimate favours the first one, the second one turns out to be around 20 times faster in practice.

\subsection{Least Common Ancestor-based algorithm}

To compute the symmetric difference we need to determine the intersection of two cycles. This can be achieved by computing Least Common Ancestors (LCA, also called Nearest Common Ancestors). Given a spanning tree $T$ of $G$, a root $r$, and two vertices $u, v \in V$, the LCA of $u, v$ is the first common vertex on the unique paths on $T,\langle u, r\rangle$ from $u$ to $r$ and $\langle v, r\rangle$ from $v$ to $r$. There exists a LCA query algorithm which takes amortized $O\left(\log ^{2} n\right)$ time for dynamically updating rooted spanning trees with $n$ vertices [28]. The output of the LCA query algorithm shall be denoted by $\operatorname{LCA}(u, v, T, r)$.

The following elementary lemma is stated without proof.

Lemma 3 Let $e=\{u, v\}$ be a chord and $r$ any root of $T$; let $p$ be the unique path in $T$ between $u$ and $v$. Then $p$ contains a unique least common ancestor $x$ of $u, v$ with respect to the root $r$ of $T$.

The procedure FCBCost, which is described in Algorithm 2, cycles over the chords of $T$ and computes the cost of the corresponding fundamental cycles by using LCAs. It relies on the following observation, which is implied by Lemma 3 .

Lemma 4 Let $e=\{u, v\}$ be a chord and $r$ a vertex of $T$. If $p_{1}=\langle u, r\rangle$ and $p_{2}=\langle v, r\rangle$ are the unique paths in $T$ between respectively $u$ and $r$, and $v$ and $r$, then $\gamma_{T}^{e}=p_{1} \triangle p_{2} \cup\{e\}$. 


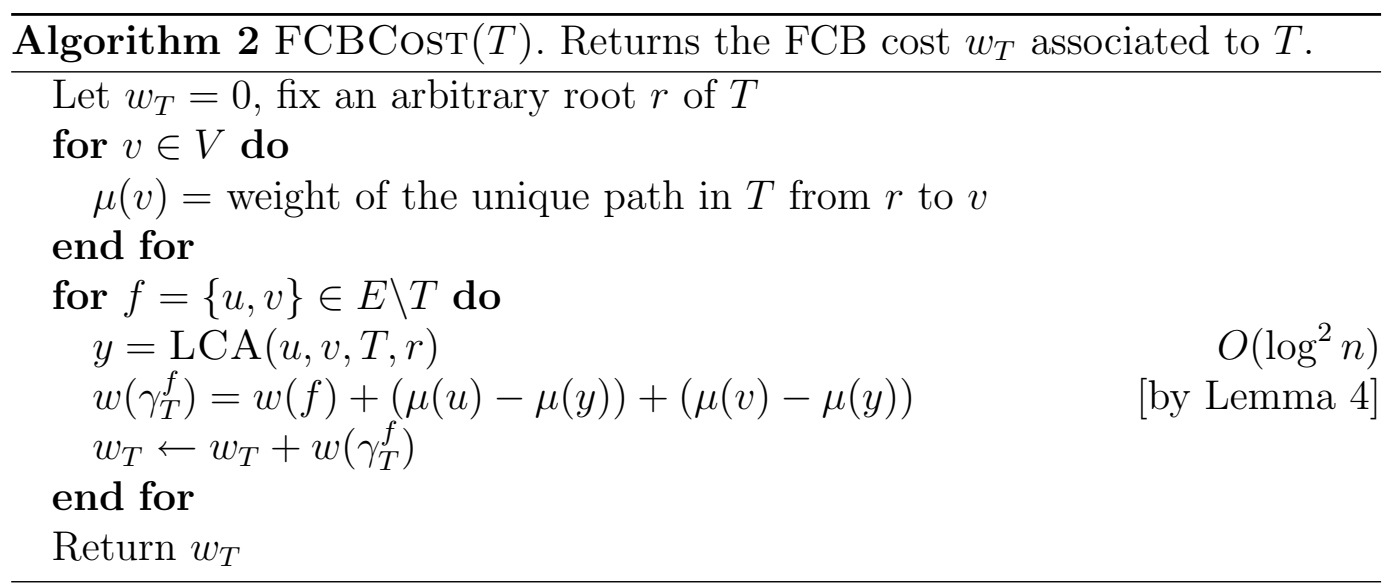

Due to the loop over $f$ and the LCA computation, the complexity of Algorithm 2 is $O\left(m \log ^{2} n\right)$.

The set $\gamma_{T}^{e} \cap \gamma_{T}^{f}$ in (1) can be computed by using LCAs.

Proposition 5 Let $\pi=(e, b)$ be an edge swap with $e=\{u, v\}$ a chord and $b$ a branch of $\gamma_{T}^{e}$, and let $f=\{t, z\} \neq e$ be another chord of $T$. Let $x, y$ be the $L C A s$ of $t, z$ with respect to the rootings $u$, and respectively $v$, of $T$. Let $p$ be the unique path in $T$ between $x$ and $y$. Then $\gamma_{T}^{e} \cap \gamma_{T}^{f}=p$.

Proof. Let $x^{\prime}$ be the LCA of $u, v$ w.r.t. the rooting $t$, and $y^{\prime}$ the LCA of $u, v$ w.r.t. the rooting $z$ of $T$. For $v_{1}, v_{2}$ distinct vertices in $V$, we indicate by $\left\langle v_{1}, v_{2}\right\rangle$ the unique path in $T$ from $v_{1}$ to $v_{2}$. Then by definition of LCA we have: $\langle x, y\rangle \subseteq T,\left\langle x^{\prime}, y^{\prime}\right\rangle \subseteq T,\langle x, u\rangle \subseteq T,\left\langle u, x^{\prime}\right\rangle \subseteq T,\langle y, v\rangle \subseteq T,\left\langle v, y^{\prime}\right\rangle \subseteq T$. Suppose $x \neq x^{\prime}$ or $y \neq y^{\prime}$. Putting the above paths together, we find that $\left(x, y, v, y^{\prime}, x^{\prime}, u, x\right)$ is a sequence of vertices on a path in $T$. Since $x$ appears as the first and last element, this path is closed, hence $T$ is a spanning tree containing a cycle, which is a contradiction. Hence $x=x^{\prime}$ and $y=y^{\prime}$. By Lemma 3, $p$ belongs to both $\gamma_{T}^{e}$ and $\gamma_{T}^{f}$, as claimed (see Figure 4).

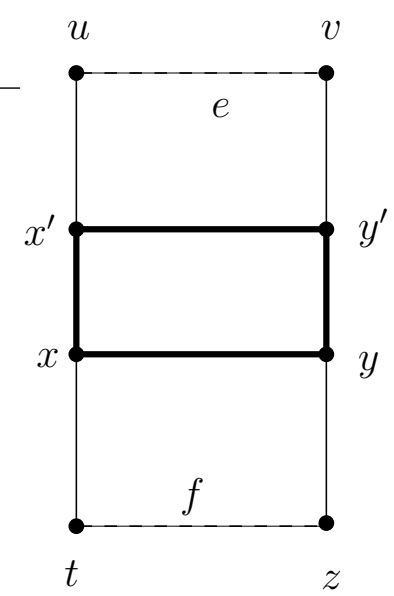

Fig. 4. Illustration of the proof of Proposition 5. 
Corollary 2 and Proposition 5 suggest the following method: given an edge swap $\pi=(e, b)$ for a chord $e$ and a branch $b$ of $\gamma_{T}^{e}$, cycle over all other chords $f \neq e$ such that $b \in \gamma_{T}^{f}$ and compute $\Delta_{\pi}$. We can then choose the edge swap leading to the best improvement. This is implemented in a slightly more efficient way in Algorithm 3 by assigning a weight $\xi$ to the vertices $u \equiv v_{1}, \ldots, v_{k}, v_{k+1} \equiv v$ in the unique path in $T$ joining the endpoints of the chord $e$. For all $i \leq k$, the weight $\sum_{j \leq i} \xi\left(v_{j}\right)$ corresponds to the change in FCB cost when $b=\left\{v_{i}, v_{i+1}\right\}$ is selected for the swapping branch in $\pi=(e, b)$.

The procedure BestSwaP is described in Algorithm 3. Its complexity is $O\left(m^{2} n\right)$, due to the nested loops over $e$ and $f$ and the path computation between $x$ and $y$.

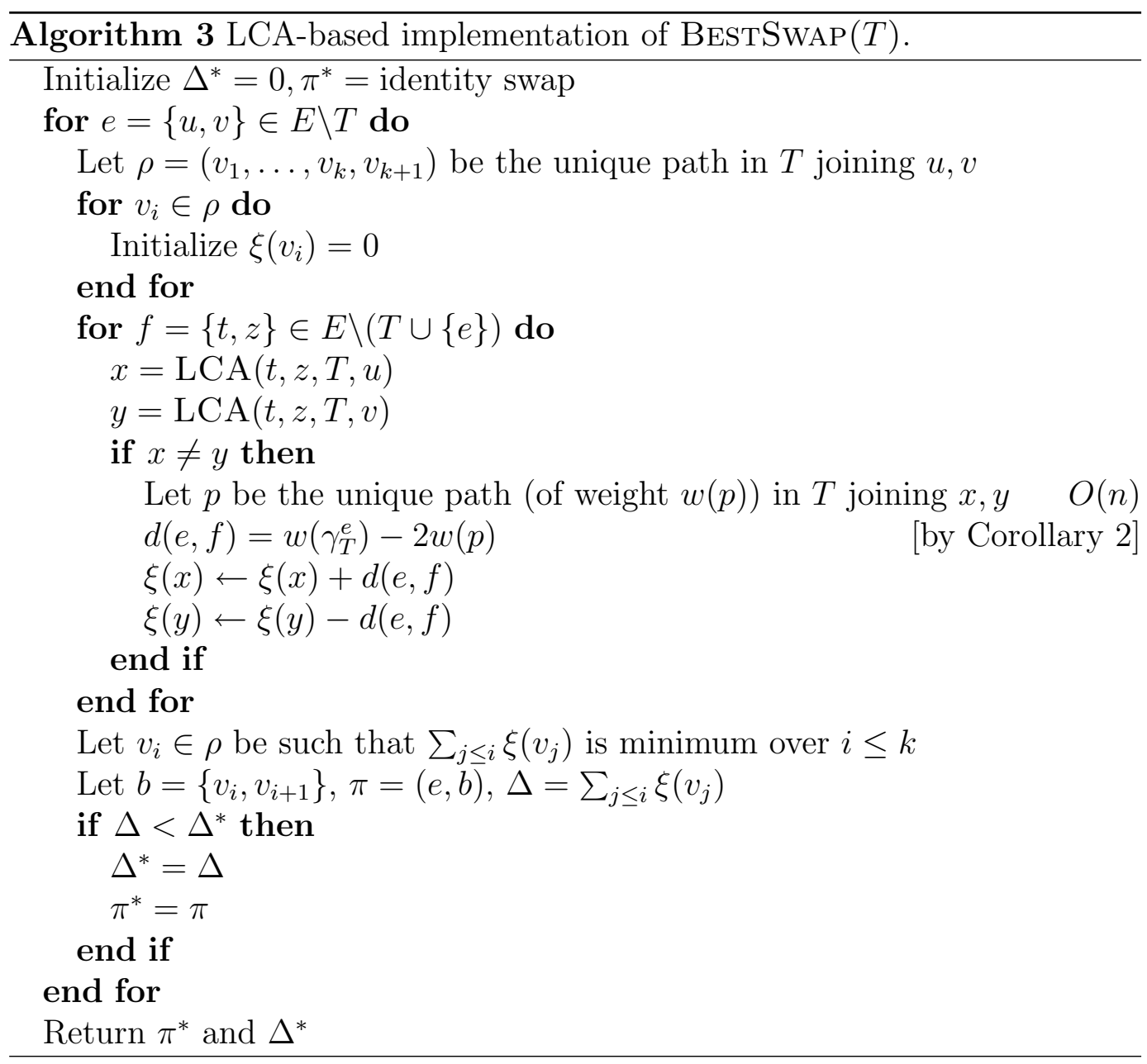

\subsection{Symmetric difference-based algorithm}

This implementation of BESTSwAP is conceptually simpler than the LCAbased one. We maintain lists of fundamental cuts and cycles associated to the 
current spanning tree, and use them to compute the cost of each edge swap (see Alg. 4). Since applying an edge swap to a spanning tree may change the fundamental cycle and cut structures considerably, efficient procedures are needed to compute $\Delta_{\pi}$ and update the fundamental cut and cycle lists. We show that any edge swap $\pi=(e, f)$ applied to a spanning tree $T$, where $e \in T$ and $f \in \delta_{T}^{e}$, changes a cut $\delta_{T}^{h}$ if and only if $f$ is also in $\delta_{T}^{h}$. Furthermore, $\pi\left(\delta_{T}^{h}\right)=\delta_{\pi T}^{h}$ is given by the symmetric difference $\delta_{T}^{h} \triangle \delta_{T}^{e}$. By Theorem 1 a similar statement holds for cycles. This makes it easy to maintain fundamental cut and cycle data structures that can be updated efficiently, by performing symmetric differences of edge sets, when $\pi$ is applied to $T$.

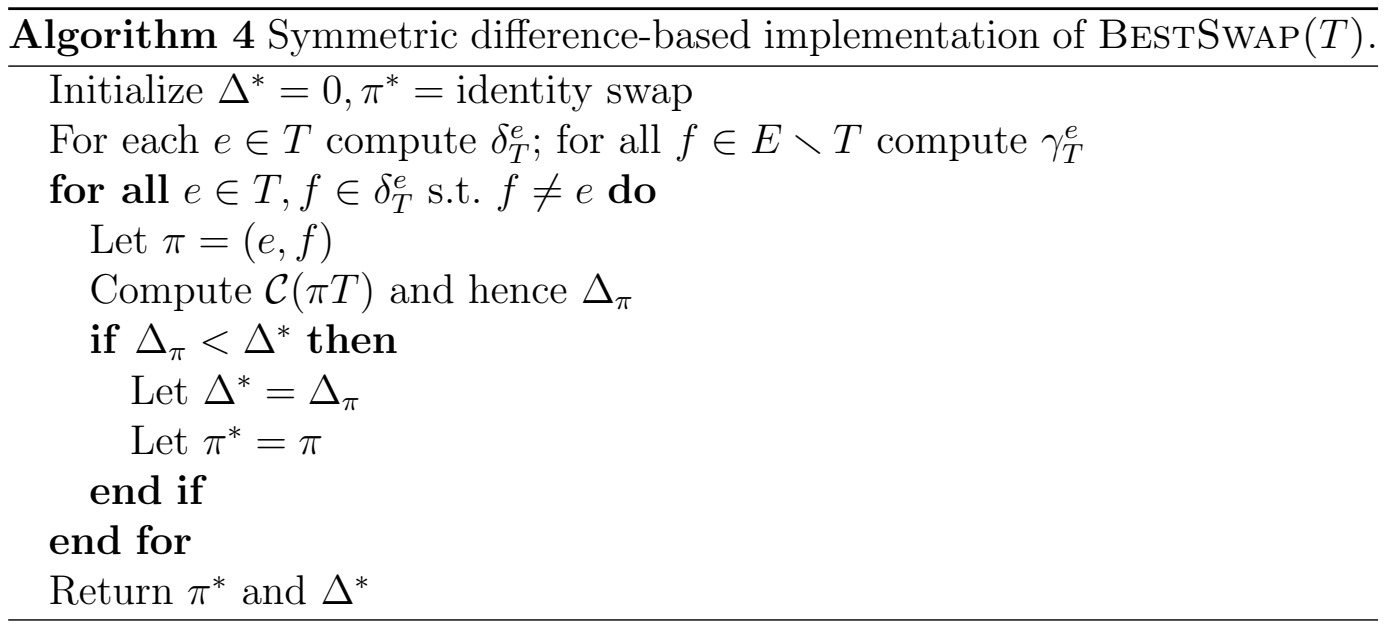

For each pair of nodes $u, v \in V,\langle u, v\rangle$ denotes the unique path in $T$ from $u$ to $v$. Let $e=\left\{u_{e}, v_{e}\right\} \in T$ be an edge of the spanning tree and $c=\left\{u_{c}, v_{c}\right\} \notin T$ be a chord. Let $p_{1}(e, c)=\left\langle u_{e}, u_{c}\right\rangle, p_{2}(e, c)=\left\langle u_{e}, v_{c}\right\rangle, p_{3}(e, c)=\left\langle v_{e}, u_{c}\right\rangle, p_{4}(e, c)=$ $\left\langle v_{e}, v_{c}\right\rangle$ and $P_{T}(e, c)=\left\{p_{i}(e, c) \mid i=1, \ldots, 4\right\}$. Note that exactly two paths in $P_{T}(e, c)$ do not contain $e$. Let $\bar{P}_{T}(e, c)$ denote the subset of $P_{T}(e, c)$ composed of those two paths not containing $e$. Let $P_{T}^{*}(e, c)$ be whichever of the sets $\left\{p_{1}(e, c), p_{4}(e, c)\right\},\left\{p_{2}(e, c), p_{3}(e, c)\right\}$ has shortest total path length in $T$, or the first one to break ties (see Figure 5). In the sequel, with a slight abuse of notation, we shall sometimes say that an edge belongs to a set of nodes, meaning that its endpoints belong to that set of nodes. For a path $p$ and a node set $N \subseteq V(G)$ we say $p \subseteq N$ if the edges of $p$ are in the edge set $E\left(G_{N}\right)$ (i.e., the edges of the subgraph of $G$ induced by $N$ ). Furthermore, we shall say that a path connects two edges $e, f$ if it connects an endpoint of $e$ to an endpoint of $f$.

The following lemma implies that the shortest paths from the endpoints of $e$ to the endpoints of $c$ do not contain $e$.

Lemma 6 For any branch $e \in T$ and chord $c \in E \backslash T$, we have $c \in \delta_{T}^{e}$ if and only if $\bar{P}_{T}(e, c)=P_{T}^{*}(e, c)$, that is e $\notin P_{T}^{*}(e, c)$.

Proof. First assume that $c \in \delta_{T}^{e}$. Denoting by $u_{c}, v_{c}$ the endpoints of $c$ and by 

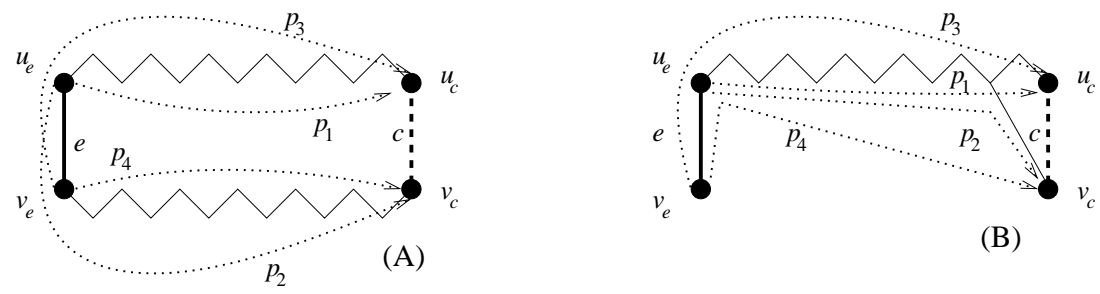

(B)

Fig. 5. (A) If $c$ is in the fundamental cut induced by $e, \bar{P}_{T}(e, c)=P_{T}^{*}(e, c)=\left\{p_{1}, p_{4}\right\}$. Otherwise, up to symmetries, we have the situation depicted in (B) where $\bar{P}_{T}(e, c) \neq P_{T}^{*}(e, c)$.

$u_{e}, v_{e}$ those of $e$, we can assume w.l.o.g. that $u_{c}, v_{c}, u_{e}, v_{e}$ are labeled so that $u_{c}, u_{e} \in S_{T}^{e}$ and $v_{c}, v_{e} \in \bar{S}_{T}^{e}$. Since there is a unique path $q$ in $T$ connecting $u_{c}$ to $v_{c}$, then $e \in q$. Thus, there are unique sub-paths $q_{1}, q_{2}$ of $q$ such that $q_{1}=\left\langle u_{e}, u_{c}\right\rangle, q_{2}=\left\langle v_{e}, v_{c}\right\rangle$ and $q_{1} \subseteq S_{T}^{e}, q_{2} \subseteq \bar{S}_{T}^{e}$ (i.e. neither $q_{1}$ nor $q_{2}$ contains $e)$. Hence $P_{T}^{*}(e, c)=\left\{q_{1}, q_{2}\right\}=\bar{P}_{T}(e, c)$. Conversely, let $P_{T}^{*}(e, c)=\left\{q_{1}, q_{2}\right\}$, and assume that $e$ is not in $q_{1}$ nor in $q_{2}$. Since either $q_{1} \subseteq S_{T}^{e}$ and $q_{2} \subseteq \bar{S}_{T}^{e}$ or vice versa, the endpoints of $c$ are separated by the cut $\delta_{T}^{e}$, i.e., $c \in \delta_{T}^{e}$.

Let $\pi=(e, f)$ be an edge swap with $e \in T, f \in \delta_{T}^{e}$ and $f \neq e$. Note that $f \in E \backslash T$. First we note that the cut in $G$ induced by $e$ with respect to $T$ is the same as the cut induced by $f$ with respect to $\pi T$.

Proposition 7 For any $\pi=(e, f)$ with $e \in T$ and $f \in \delta_{T}^{e}$ such that $f \neq e$, we have $\pi\left(\delta_{T}^{e}\right)=\delta_{\pi T}^{f}$.

Proof. Since $f \in \delta_{T}^{e}$, swapping $e$ with $f$ does not modify the partitions that induce the cuts, i.e., $S_{T}^{e}=S_{\pi T}^{f}$.

Second, we show that the cuts that do not contain $f$ are not affected by $\pi$.

Proposition 8 For each $h \in T$ such that $h \neq e$, and $f \notin \delta_{T}^{h}$, we have $\pi\left(\delta_{T}^{h}\right)=$ $\delta_{T}^{h}$.

Proof. Let $g \in \delta_{T}^{h}$. By Lemma 6, the shortest paths $p_{1}^{T}, p_{2}^{T}$ from the endpoints of $h$ to the endpoints of $g$ do not contain $h$. We shall consider three possibilities.

(1) In the case where $e$ and $f$ do not belong either to $p_{1}^{T}$ or $p_{2}^{T}$ we obtain trivially that $\bar{P}_{\pi T}(f, e)=\bar{P}_{T}(e, f)=P_{T}^{*}(e, f)=P_{\pi T}^{*}(f, e)$ and hence the result.

(2) Assume now that $e \in p_{1}^{T}$, and that both $e, f$ are in $S_{T}^{h}$. The permutation $\pi$ changes $p_{1}^{T}$ so that $f \in p_{1}^{\pi T}$, whilst $p_{2}^{\pi T}=p_{2}^{T}$. Now $p_{1}^{\pi T}$ is shortest because it is the unique path in $\pi T$ connecting the endpoints of $p_{1}^{T}$, and since $h \notin p_{1}^{\pi T}, p_{2}^{\pi T}$ because $\pi$ does not affect $h$, we obtain $\bar{P}_{\pi T}(e, c)=P_{\pi T}^{*}(e, c)$. 
(3) Suppose that $e \in p_{1}^{T}, e \in S_{T}^{h}$ and $f \in \bar{S}_{T}^{h}$. Since $f \in \delta_{T}^{e}$, by Lemma 6 there are shortest paths $q_{1}^{T}, q_{2}^{T}$ connecting the endpoints of $e$ and $f$ such that $q_{1}^{T} \subseteq S_{T}^{e}, q_{2}^{T} \subseteq \bar{S}_{T}^{e}$. Since $e \in S_{T}^{h}, f \in \bar{S}_{T}^{h}$ and $T$ is tree, there is an $i$ in $\{1,2\}$ such that $h \in q_{i}^{T}$ (assume w.l.o.g. that $i=1$ ), so $q_{1}^{T}=r_{1}^{T} \cup\{h\} \cup r_{2}^{T}$ where $r_{1}^{T} \subseteq S_{T}^{h}$ connects $h$ and $e$, and $r_{2}^{T} \subseteq \bar{S}_{T}^{h}$ connects $h$ and $f$. If we let $q^{T}=r_{1}^{T} \cup\{e\} \cup q_{2}^{T}$, then $q^{T}$ is the unique path in $S_{T}^{h}$ connecting $h$ and $f$. Since $r_{2}^{T}$ connects $h$ and $f$ in $\bar{S}_{T}^{h}$, we must conclude that $f \in \delta_{T}^{h}$, which is a contradiction.

Third, we prove that any cut containing $f$ is mapped by the edge swap $\pi=$ $(e, f)$ into its symmetric difference with the cut induced by $e$ of $T$.

Theorem 9 For each $h \in T$ such that $h \neq e$ and $f \in \delta_{T}^{h}$, we have $\pi\left(\delta_{T}^{h}\right)=$ $\delta_{T}^{h} \triangle \delta_{T}^{e}$.

The proof is lengthy and hence is given in Appendix A.

\subsection{Complexity comparison}

It was shown in Section 2.3 that the complexity of the BESTSwAP procedure based on LCA computation is $O\left(m^{2} n\right)$.

In the alternative algorithm of Section 2.4, fundamental cuts and cycles are determined and updated by using symmetric differences of edge sets, which require linear time in the size of the sets. Since for any spanning tree $T$ there are $m-n+1$ fundamental cycles with at most $n$ edges, and $n-1$ fundamental cuts with at most $m$ edges, updating the fundamental cut and cycle structures after the application of an edge swap $(e, f)$ requires $O(m n)$. Doing this for each branch of the tree and for each chord in the fundamental cut induced by the branch, leads to a $O\left(m^{2} n^{2}\right)$ worst-case complexity, apparently leading to a worse algorithm.

Running the implementation of both algorithms, however, showed a consistent performance difference in favour of the second algorithm. More precisely, tested on a set of 60 instances of various types (see the table in Appendix B), the LCA-based algorithm was on average around 20 times slower than the symmetric difference-based one (with a standard deviation of around 10); profiling the code suggested that this was essentially due to the algorithm rather than the implementation. An intuitive explanation may be found in the fact that worst-case complexity analysis may not accurately reflect the performance of the second algorithm. Take for instance the update of the fundamental cuts: there is a first loop on the $n-1$ tree branches inducing the cuts, and a second nested loop of $O(m)$ chords in each cut. This eventually leads 
to the (apparently) very costly $O\left(m^{2} n^{2}\right)$ term. On average, however, each cut is very far from attaining the worst-case estimation of $m$ edges. Instead, one should consider the total number of edges counted jointly by the two nested loops, which is usually much lower than the worst-case $m(n-1)$.

In support of the above view, the following simple average-case observations can be made for unweighted graphs.

(1) Let $\bar{k}$ be the average cardinality of each cycle in the minimum FCB: then $\bar{k}=\frac{f^{*}}{m-n+1}$ where $f^{*}$ is the cost of the minimum FCB.

(2) Each chord belongs on average to the $\bar{k}-1$ cuts induced by its own cycle.

(3) The average length of each cut is therefore $\frac{m(\bar{k}-1)}{n-1}$, and the total number of edges counted jointly by the two nested loops is on average $m(\bar{k}-1)$.

(4) Asymptotically, $m(\bar{k}-1)$ is $O\left(f^{*}\right)$.

It follows that, for unweighted graphs, the cost of the minimum FCB is a reasonable estimate of the complexity cost of each swap. Since the main focus of this work is experimental, in the sequel we only report the computational results for the faster algorithm based on symmetric differences.

Finally, we remark that the computing time taken for a single iteration of the local search heuristic (i.e. an application of the BestSwap algorithm) is (asymptotically) comparable with the time taken for constructing the initial solution (see e.g. worst-case complexity bounds in [26]). This suggests that such heuristics are not well-suited for real-time or time-critical applications.

\section{Metaheuristics}

To go beyond the scope of local search and try to escape from local minima, we have implemented and tested two well-known metaheuristics: variable neighborhood search (VNS) [29] and tabu search (TS) [30].

\subsection{Variable neighborhood search}

VNS is a relatively recent metaheuristic which relies on iteratively exploring neighborhoods of growing size to identify better local optima [29]. More precisely, in VNS one attempts to escape from a local minimum $x^{\prime}$ by choosing another random starting point in increasingly larger neighborhoods of $x^{\prime}$. If the cost of the local minimum $x^{\prime \prime}$ obtained by applying the local search from $x^{\prime}$ is smaller than the cost of $x^{\prime}$, then $x^{\prime \prime}$ becomes the new best local minimum and the neighborhood size is reset to its minimal value. This procedure 
is repeated until a given termination condition is met. VNS was successfully tested on many combinatorial and continuous optimization problems [31].

For the Min FCB problem, given a locally optimal spanning tree $T^{\prime}$ provided by the local search algorithm, we consider a neighborhood of size $p$ consisting of all those spanning trees $T$ that can be reached from $T^{\prime}$ by applying $p$ consecutive edge swaps. A random solution in a neighborhood of size $p$ is obtained by generating a sequence of $p$ random edge swaps and applying it to $T^{\prime}$. The algorithm is summarized in Figure 6.

For the whole test set, the parameters were set as follows: minimum neighborhood size $k=2$ ( $k=1$ would simply explore the local search neighborhood again), maximum neighborhood size $K=5$, number of trials in each neighborhood $F=1$.

(1) Let $T^{*}$ be a locally minimal spanning tree found with LS, and $\varphi^{*}$ its FCB cost

(2) Initialize minimum and maximum neighborhood sizes $k, K$ and number $F$ of local searches in each neighborhood

(3) Set $p=k$ and $T=T^{*}$

(4) for all $i \leq F$ do

for all $j \leq p$ do

Choose random edge swap $\pi=(b, c)$

$T=\pi T$

Let $T^{\prime}=\operatorname{LS}(T)$ and $\varphi^{\prime}$ be the FCB cost of $T^{\prime}$

if $\varphi^{\prime}<\varphi^{*}$ then let $T^{*}=T^{\prime}, \varphi^{*}=\varphi^{\prime}$ and go to step (3)

(5) $p \leftarrow p+1$

(6) if $p>K$ then terminate else go to step (4)

Fig. 6. The VNS algorithm for the Min FCB problem. In step 4, LS $(T)$ denotes the spanning tree provided by the local search algorithm.

\subsection{Tabu search}

Our implementation of TS [30] includes diversification steps "à la VNS" (vTS). In order to escape from local minima, a best available edge swap is applied to the current solution (even if it worsens the FCB cost) and is inserted in a tabu list. If all possible edge swaps are tabu or a pre-determined number $S$ of successive non-improving moves is exceeded, $t$ random edge swaps are applied to the current spanning tree; this move is called a random shaker of size $t$. The number $t$ increases until a limit $K$ is reached, and is then re-set to a starting size $k$. The procedure runs until a given termination condition is met. The algorithm is summarized in Figure 7. 
For the whole test set, we took: maximum tabu list size $\Lambda=10$, minimum and maximum random shaker sizes $k=2, K=30$, non-improving moves limit $S=20$.

(1) Let $T^{*}$ be a locally minimal spanning tree found with LS, and $\varphi^{*}$ its FCB cost

(2) Initialize tabu list length $\Lambda$, minimum and maximum random shaker size $k, K$, non-improving moves limit $S$

(3) Initialize FIFO tabu list $L=\emptyset$, cost-increasing edge swap counter $s=0$, random shaker size $p=k$

(4) Choose a cost-increasing edge swap $\pi \neq 1$ s.t. cost increase is minimal and $\pi \notin L$; let $s=s+1$

(5) if such a $\pi$ does not exist, or $s>S$ then let $\pi$ be a random shaker of size $p$; set $p=p+1$ and $s=0$

(6) if $p>K$ then terminate

(7) Let $T=\pi T^{*}$ and $L=L \cup\left\{\pi^{-1}\right\}$

(8) if $|L|>\Lambda$ then remove oldest edge swap from $L$

(9) Let $T^{\prime}=\operatorname{LS}(T)$ and $\varphi^{\prime}$ be the FCB cost of $T^{\prime}$

(10) if $\varphi^{\prime}<\varphi^{*}$ then let $\varphi^{*}=\varphi^{\prime}, T^{*}=T^{\prime}, s=0, p=k$

(11) Go to step (4)

Fig. 7. The TS algorithm for the Min FCB problem. In step $7, \pi^{-1}$ indicates the inverse edge swap; in step $4, \mathrm{LS}(T)$ denotes the spanning tree provided by the local search algorithm.

Other TS variants were tested. In particular, we implemented a "pure" TS (pTS) with no diversification, and a fine-grained TS (fTS) where, instead of forbidding moves (edge swaps), feasible solutions are forbidden by exploiting the fact that spanning trees can be stored in a very compact form. We also implemented a TS variant with the above-mentioned diversification steps where pTS tabu moves and fTS tabu solutions are alternatively considered. Although the results are comparable on most test instances, vTS performs best on average. Computational experiments indicate that diversification is more important than intensification when searching the MiN FCB solution space with our type of edge swaps. Variable length tabu lists were also tried, but the results were similar to those obtained with fixed-length lists.

\section{Lower bounds}

Lower bounds on the cost of the optimal solutions are useful to assess the performance of heuristics. The linear relaxations of three different mixed integer programming formulations were discussed in [32]. In this section, we describe an improved formulation that uses non-simultaneous flows on arcs to en- 
sure that the cycle basis is fundamental. Consider an edge-biconnected graph $G=(V, E)$ with a non-negative cost $w_{i j}$ assigned to each edge $\{i, j\} \in E$. For each node $v \in V, \delta(v)$ denotes the node star of $v$, i.e., the set of all edges incident to $v$. Let $G_{0}=(V, A)$ be the directed graph associated with $G$, namely $A=\{(i, j),(j, i) \mid\{i, j\} \in E\}$. We use two sets of decision variables. For each edge $\{k, l\} \in E$, the variable $x_{i j}^{k l} \geq 0$ represents the flow through arc $(i, j) \in A$ from $k$ to $l$. Moreover, for each edge $\{i, j\} \in E$, the variable $z_{i j}$ is equal to 1 if edge $\{i, j\}$ is in the spanning tree of $G$, and equal to 0 otherwise. For each pair of $\operatorname{arcs}(i, j) \in A$ and $(j, i) \in A$, we define $w_{j i}=w_{i j}$. The following MIP formulation for the MiN FCB problem provides much tighter bounds than those considered in [32]:

$$
\begin{aligned}
\min \quad \sum_{\{k, l\} \in E} \sum_{(i, j) \in A} w_{i j} x_{i j}^{k l}+\sum_{\{i, j\} \in E} w_{i j}\left(1-2 z_{i j}\right) \\
\text { s.t. } \quad \sum_{j \in \delta(k)}\left(x_{k j}^{k l}-x_{j k}^{k l}\right)=1 \quad \forall\{k, l\} \in E \\
\sum_{j \in \delta(i)}\left(x_{i j}^{k l}-x_{j i}^{k l}\right)=0 \quad \forall\{k, l\} \in E, \forall i \in V \backslash\{k, l\} \\
x_{i j}^{k l} \leq z_{i j} \quad \forall\{k, l\} \in E, \forall\{i, j\} \in E \\
x_{j i}^{k l} \leq z_{i j} \quad \forall\{k, l\} \in E, \forall\{i, j\} \in E \\
\sum_{\{i, j\} \in E} z_{i j}=n-1 \\
x_{i j}^{k l} \geq 0 \quad \forall\{k, l\} \in E, \forall(i, j) \in A \\
z_{i j} \in\{0,1\} \quad \forall\{i, j\} \in E .
\end{aligned}
$$

For each edge $\{k, l\} \in E$, a path $p$ from $k$ to $l$ is represented by a unit of flow through each arc $(i, j)$ in $p$. In other words, a unit of flow exits node $k$ and enters node $l$ after going through all other (possible) nodes in $p$. For each edge $\{k, l\} \in E$, the flow balance constraints (3) and (4) account for a directed path connecting nodes $k$ and $l$. Note that the flow balance constraint for node $l$ is implied by constraints (3) and (4). Since constraints (5) and (6) require that $z_{i j}=1$ for every edge $\{i, j\}$ contained in some path (namely with a strictly positive flow), the $z$ variables define a connected subgraph of $G$. Finally, constraint (7) ensures that the connected subgraph defined by the $z$ variables is a spanning tree. The objective function (2) adds the cost of the path associated to every edge $\{k, l\} \in E$ and the cost of all tree chords, and subtracts from it the cost of the tree branches (which are counted when considering the path for every edge $\{k, l\}$ ). The main shortcoming of this formulation is that it contains a large number of variables and constraints and hence it is hard to solve to optimality even for medium-sized instances.

The bounds reported in the next section were obtained by letting CPLEX 
MIP solver (v. 9.1, [33]) process the root node of the branch-and-bound tree, with automatic cut generation enabled. For small instances, the solver was run to completion.

\subsection{Efforts towards tighter lower bounds}

A substantial effort was undertaken in order to obtain tighter lower bounds but unfortunately to no avail. A number of different ILP formulations and corresponding Lagrangian relaxations of some of the constraints were considered. Almost invariably, one of the two resulting subproblems was solvable in polynomial time and the other was computationally as difficult as (or more than) solving the MiN FCB problem itself. When two easy subproblems were obtained, the resulting bounds were weaker or the same as those obtained by solving the linear relaxation of the formulation given above.

One of the most (apparently) promising Lagrangian relaxations we considered is the following "partial" ILP formulation:

$$
\begin{aligned}
& \min z= \sum_{i \in E} \sum_{k=1}^{\nu} w_{i} x_{i k} \\
& \text { s.t. } \quad \sum_{k=1}^{\nu} x_{i k} \geq 1 \quad \forall i \in E \\
&\left\{i \mid y_{i}=1\right\} \quad \text { is a spanning tree of } \mathrm{G} \\
& \sum_{k=1}^{\nu} x_{i k} \leq 1+\nu y_{i} \forall i \in E \\
& \sum_{i \in \delta_{j}} x_{i k}=2 s_{j k} \quad \forall j \in V, k \leq \nu \\
& \sum_{i \in E} x_{i k} \geq 3 \quad \forall k \leq \nu .
\end{aligned}
$$

where:

$$
\begin{array}{rlrl}
x_{i k} & =1 \quad \text { if edge } i \in \text { cycle } k & & \text { and } 0 \text { otherwise } \\
y_{i} & =1 \text { if edge } i \in \text { spanning tree } T & \text { and } 0 \text { otherwise } \\
s_{j k} & =1 \quad \text { if vertex } j \in \text { cycle } k & & \text { and } 0 \text { otherwise }
\end{array}
$$

and $\delta_{j}$ is the star of vertex $j$ (there are $\Theta(m n)$ variables in (8)-(13), the same as in (2)-(7)). The only "complicating constraints" of this formulation, involving both the $x$ and $y$ variables, are those in Equation (11). By relaxing these constraints we can decompose the MIN FCB problem into two separate (simpler) problems. We relax the $|E|$ constraints (11) using Lagrange multipliers 
$\lambda_{i}$ for all $i \in E$ to obtain:

$$
\begin{aligned}
z(x, y, \lambda) & =\sum_{i \in E} w_{i} \sum_{k=1}^{\nu} x_{i k}+\sum_{i \in E} \lambda_{i}\left(\sum_{k=1}^{\nu} x_{i k}-1-\nu y_{i}\right)= \\
& =\sum_{i \in E}\left(w_{i}+\lambda_{i}\right) \sum_{k=1}^{\nu} x_{i k}-\nu \sum_{i \in E} \lambda_{i} y_{i}-\sum_{i \in E} \lambda_{i}= \\
& =z_{1}-z_{2}-z_{3}
\end{aligned}
$$

where

$$
\begin{aligned}
z_{1}(x, \lambda) & =\sum_{i \in E}\left(w_{i}+\lambda_{i}\right) \sum_{k=1}^{\nu} x_{i k} \\
z_{2}(y, \lambda) & =\nu \sum_{i \in E} \lambda_{i} y_{i} \\
z_{3}(\lambda) & =\sum_{i \in E} \lambda_{i} .
\end{aligned}
$$

This separates the problem into two independent subproblems: MAX SPANNING TREE ( $y$ variables, objective function $z_{2}$ ) and a special form of MIN Eulerian CyCle ( $x$ variables, objective function $z_{1}$ ). The MaX Spanning TREE problem is formulated as $\min _{y}\left\{z_{2}(y, \lambda) \mid(10)\right\}$ and solved combinatorially with a greedy method. The special Min EuLERIAN CYCLE problem is formulated as $\min _{x, s}\left\{z_{1}(x, s, \lambda) \mid(9) \wedge(12) \wedge(13)\right\}$ and also solved combinatorially (we omit the details). The Lagrangian dual problem is therefore

$$
\max _{\lambda \geq 0}\left(\min _{x, s}\left\{z_{1}(x, \lambda) \mid(9) \wedge(12) \wedge(13)\right\}-\min _{y}\left\{z_{2}(y, \lambda) \mid(10)\right\}-z_{3}(\lambda)\right),
$$

which we tried to solve using a subgradient method [34], but to no avail, getting a bound that was identical to the relaxation of the MIN FCB formulation (2)(7).

Since the constraints in the MIN FCB formulation are the same as in the minimum routing cost tree formulation, we tried to tighten the bound by adding valid cuts used in [23]. In particular, we considered the cardinality cuts stating that each subtree must contain a number of edges equal to the number of nodes minus one. However, the solution of the linear relaxation of the above formulation, albeit fractional, already satisfied all these cuts on all tested instances.

For large instances, even solving the linear relaxation of our formulation is a very challenging task. In those cases, a lower bound was obtained by finding the minimum (non-fundamental) cycle basis of the graph using an improved version of Horton's algorithm $[16,18,35]$. On average, this is a very weak lower bound, whose difference with respect to the optimal FCB cost grows with the 
size of the instance. In the case of mesh graphs, we established experimentally that the gap between this lower bound and the heuristic solution increases about linearly with number of vertices in the graph (in the case of unweighted graphs, Thm. 6.6 of [38] gives an asymptotic $\frac{1}{1024} \ln (n)$ lower bound for square grids). Further information about bounds and asymptotic sizes for mesh grids can be found in [36] and the references cited therein.

\section{Computational results}

Our edge-swapping local search algorithm and metaheuristics were implemented in $\mathrm{C}++$ and tested on various types of unweighted and weighted graphs. CPU times refer to a Pentium $42.66 \mathrm{GHz}$ processor with $1 \mathrm{~GB}$ RAM running Linux. We considered the following classes of instances:

- star graphs and rectangular mesh graphs with known global optima,

- square mesh graphs with unit costs on the edges,

- Euclidean graphs generated randomly, with weights proportional to the edge lengths,

- two instances taken from a real-life periodic timetabling problem,

- one instance taken from a real-life electrical circuit testing application,

- 2D and 3D toroidal graphs with unit costs on the edges.

Some instances are from the literature, while others were generated on purpose. In the next subsections, we provide the corresponding information and report the results obtained for each one of them. Each instance was tested with the following heuristics: the local search algorithm (LS), the NT-heuristic cited in [4], and the VNS and TS algorithms described in Section 3 (which were run for 10 minutes after finding the first local optimum, unless specified otherwise). For most instances, we also report a lower bound.

\subsection{Graphs with known optima}

$N$-Star graphs. These graphs can be represented as regular polygons having $N$ sides. The nodes are those adjacent to the $N$ sides and a node at the center of the polygon; the edges are the $N$ sides and all edges connecting the central node with each of the other nodes. An example with $N=8$ sides is shown in Figure 8(a). The spanning tree giving rise to the optimal FCB consists of all edges connecting the central node with each of the other nodes. All the cycles in the optimal FCB have 3 edges; each of the $N$ sides of the polygon is a chord. The optimal FCB cost is $3 N$. We considered the instances for all $N$ up to 50, and in all cases the optimal solution was reached without any edge 
swap, i.e., the initial constructive heuristic always found the optimal solution.

Rectangular mesh graphs. These graphs consist of a rectangular array of $4 \times N$ nodes; Figure 8(b) shows an example with $N=9$, together with the optimal solution. Although it is easy to show that the optimal solutions for such instances are all similar to that given in Figure 8(b), constructive heuristics fail to find it. The optimal FCB has $2(n-3)+6$ square cycles (4 edges each) and $N-3$ rectangular cycles (6 edges each) with an optimal cost of $14 N-28$. All instances from $N=10$ to $N=100$ were tried, and the local search heuristic always managed to find the optimum, either within 2 or 4 edge swaps.

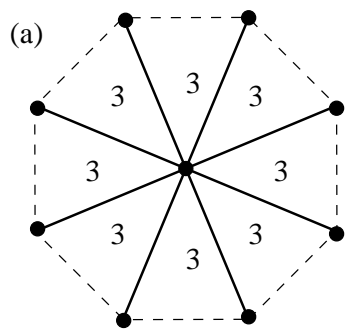

(b)

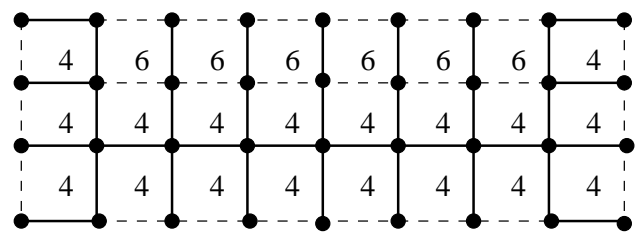

Fig. 8. Instance of 8-star graph with optimal FCB (a). Instance of $(4 \times 9)$-rectangular mesh with optimal FCB (b).

\subsection{Unweighted mesh graphs}

One of the most challenging testbeds for the Min FCB problem is given by the square $N \times N$ mesh graphs with unit costs on the edges. Due to the large number of symmetries in these graphs, there are many different spanning trees with identical associated FCB costs. Uniform cost square mesh graphs have $n=N^{2}$ nodes and $m=2 N(N-1)$ edges. Table 1 reports the FCB costs and corresponding CPU times. The lower bounds for this class of graphs correspond to the formula $4(m-n+1)$, namely to the cost of a minimum non-fundamental cycle basis. In the case of mesh graphs, it is also a minimum weakly fundamental cycle basis.

For this class of graphs LS finds much better solutions than NT, but the differences in CPU times are enormous. TS performs very slightly better than VNS on average.

Since these results were first presented in [1], considerable attention has been devoted to the Min FCB problem. In particular, the focus in [36] is on mesh graphs, and the authors improve the FCB costs by around $16 \%$ with respect to Table 1 and the lower bounds by around 67\%. Our methods, of course, are applicable to general graphs, whereas those developed in [36] are tailored to mesh graphs. In any case, it appears clear that reducing the gap between lower and upper bounds is a definite challenge for future research. 
Table 1

\begin{tabular}{|c||cc|cc|c|c|c|}
\hline \multicolumn{1}{|c||}{} & \multicolumn{2}{c|}{ LS } & \multicolumn{2}{c|}{ NT } & VNS & TS & Bound \\
\hline$N$ & Cost & Time & Cost & Time & Cost & Cost & Cost \\
\hline 5 & $72^{*}$ & $0: 00: 00$ & 78 & $0: 00: 00$ & 72 & 72 & 64 \\
10 & 474 & $0: 00: 00$ & 518 & $0: 00: 00$ & $466^{*}$ & $466^{*}$ & 324 \\
15 & 1318 & $0: 00: 00$ & 1588 & $0: 00: 00$ & 1280 & $1276^{*}$ & 784 \\
20 & 2608 & $0: 00: 03$ & 3636 & $0: 00: 00$ & $2572^{*}$ & 2590 & 1444 \\
25 & 4592 & $0: 00: 16$ & 6452 & $0: 00: 00$ & 4464 & $4430^{*}$ & 2304 \\
30 & 6956 & $0: 00: 47$ & 11638 & $0: 00: 00$ & 6900 & $6882^{*}$ & 3364 \\
35 & 10012 & $0: 02: 19$ & 16776 & $0: 00: 00$ & 9982 & $9964^{*}$ & 4624 \\
40 & 13548 & $0: 06: 34$ & 28100 & $0: 00: 01$ & $13524^{*}$ & 13534 & 6084 \\
45 & $18100^{*}$ & $0: 14: 22$ & 35744 & $0: 00: 01$ & 18100 & 18100 & 7744 \\
50 & $23026^{*}$ & $0: 31: 04$ & 48254 & $0: 00: 03$ & 23026 & 23552 & 9604 \\
\hline
\end{tabular}

Computational results (CPU times in format h:mm:ss) for $N \times N$ mesh graphs. Values marked with ${ }^{*}$ denote the best value found for the instance.

\subsection{Random Euclidean graphs}

We have generated simple random edge-biconnected graphs. The nodes are positioned uniformly at random on a $20 \times 20$ square centered at the origin. Between each pair of nodes an edge is generated with probability $p$, with $0<p<1$ (also called the edge density). The cost of an edge is equal to the Euclidean distance between its adjacent nodes. For each $n$ in $\{10,20,30,40,50\}$ and $p$ in $\{0.2,0.4,0.6,0.8\}$, we have generated a random graph of size $n$ with edge probability $p$. The computational results are given in Table 2 . Lower bounds have been computed by solving a linear relaxation of the formulation in Section 4. The average percentage gap between the LS heuristic and lower bounding FCB costs is $8.19 \%$, the standard deviation is $\pm 5.15 \%$, and the mode is $6 \%$ (these values have been obtained considering a larger instance set than that reported in Table 2, namely 81 instances). It is worth pointing out that the lower bounds obtained by solving the linear relaxation of the formulation presented in Section 4 are generally much tighter than those derived from the formulations considered in [32]. The performance of VNS and TS is very similar on this class of graphs.

\subsection{Instances from real-life applications}

Periodic Timetabling. An interesting application of MIN FCB arises in periodic timetabling for transportation systems. In [5], the timetables of the Berlin underground are designed by considering a mathematical programming model based on the Periodic Event Scheduling Problem (PESP) [37] and the associated graph $G$ in which nodes correspond to events. Since the number of integer variables in the model can be minimized by identifying an FCB of $G$ and the 


\begin{tabular}{|c|c|c|c|c|c|c|c|c|}
\hline & \multicolumn{8}{|c|}{$p=0.2$} \\
\hline$n$ & LS & Time & NT & Time & VNS & $\mathrm{TS}$ & Bound & Time \\
\hline 10 & $216.698^{\dagger}$ & 0 & 222.987 & 0 & $216.698^{\dagger}$ & $216.698^{\dagger}$ & $216.698^{\dagger}$ & 0 \\
\hline 20 & $1052.38^{\dagger}$ & 0 & 1178.15 & 0 & $1052.38^{\dagger}$ & $1052.38^{\dagger}$ & $1052.38^{\dagger}$ & $0: 56$ \\
\hline 30 & 3315.89 & 0 & 3868.78 & 0 & $3111.71^{*}$ & $3311.71^{*}$ & 2750.92 & $0: 28$ \\
\hline 40 & 4634.04 & 0 & 6154.1 & 0.01 & $4504.84^{*}$ & $4505.84^{*}$ & 4065.187 & $16: 58$ \\
\hline \multirow[t]{2}{*}{50} & 7007.34 & 0:01 & 9326.82 & 0.01 & $6991.53^{*}$ & $6991.53^{*}$ & 6448.711 & $2: 38: 51$ \\
\hline & \multicolumn{8}{|c|}{$p=0.4$} \\
\hline$n$ & $\mathrm{LS}$ & Time & NT & Time & VNS & $\mathrm{TS}$ & Bound & Time \\
\hline 10 & 472.599 & 0 & 504.275 & 0 & $459.305^{\dagger}$ & $459.305^{*}$ & $459.305^{\dagger}$ & $0: 02$ \\
\hline 20 & 2021.82 & 0 & 2801.28 & 0 & $2021.37^{*}$ & $2021.37^{*}$ & 1894.747 & 0:08 \\
\hline 30 & 4467.13 & 0 & 5482.21 & 0.01 & $4455.2^{*}$ & $4455.2^{*}$ & 4265.6 & $22: 56$ \\
\hline 40 & 7685.97 & 0:01 & 9509.93 & 0.01 & $7648^{*}$ & $7648^{*}$ & - & - \\
\hline \multirow[t]{2}{*}{50} & 11096.8 & 0:05 & 15667.5 & 0.01 & $11022.8^{*}$ & $11022.8^{*}$ & - & - \\
\hline & \multicolumn{8}{|c|}{$p=0.6$} \\
\hline$n$ & LS & Time & NT & Time & VNS & $\mathrm{TS}$ & Bound & Time \\
\hline 10 & 581.525 & 0 & 593.475 & 0 & $547.406^{\dagger}$ & $547.406^{\dagger}$ & $547.406^{\dagger}$ & 0:08 \\
\hline 20 & 2776.22 & 0 & 3959.41 & 0 & $2756.6^{*}$ & $2756.6^{*}$ & 2627.558 & $0: 59$ \\
\hline 30 & 7031.2 & 0 & 8243.03 & 0.01 & 6979.15 & $6967.98^{*}$ & 6445.83 & $39: 32$ \\
\hline 40 & 11686.0 & 0:02 & 13469.5 & 0.01 & $11513^{*}$ & $11513^{*}$ & - & - \\
\hline \multirow[t]{2}{*}{50} & 19387.3 & $0: 10$ & 24440.3 & 0.02 & $19174.1^{*}$ & $19174.1^{*}$ & - & - \\
\hline & \multicolumn{8}{|c|}{$p=0.8$} \\
\hline$n$ & $\mathrm{LS}$ & Time & NT & Time & VNS & $\mathrm{TS}$ & Bound & Time \\
\hline 10 & 992.866 & 0 & 992.866 & 0 & $775.838^{\dagger}$ & $775.838^{\dagger}$ & $775.838^{\dagger}$ & $0: 26$ \\
\hline 20 & 3478.11 & 0 & 4019.34 & 0 & $3383.45^{*}$ & $3383.45^{*}$ & 3164.9 & $2: 31$ \\
\hline 30 & 8971.78 & 0:01 & 10847.8 & 0.01 & $8384.32^{*}$ & $8384.32^{*}$ & 7823.848 & $1: 43: 05$ \\
\hline 40 & 14946.4 & 0:07 & 20229.2 & 0.01 & 14870.7 & $14792^{*}$ & - & - \\
\hline 50 & 25349.9 & $0: 12$ & 28586.8 & 0.02 & $25061.2^{*}$ & 25245.5 & - & - \\
\hline
\end{tabular}

Table 2

Computational results (CPU times in seconds or hh:mm:ss) for Euclidean random graphs. Lower bound values marked with ${ }^{\dagger}$ denote an optimal solution (MIP solved to optimality by CPLEX v. 9.1 [33]). FCB costs marked with * denote the best value found for the instance. Missing values are due to excessive CPU timings.

number of discrete values that each integer variable can take is proportional to the total FCB cost, good models for the PESP problem can be obtained by finding minimum fundamental cycle bases of the corresponding graph $G$. Due to the way the edge costs are determined, the Min FCB instances arising from this application have a high degree of symmetry, which makes them difficult. The results reported in Table 3 for instance timtab2 (with 88 nodes and 316 edges), which is available from MIPLIB (http://miplib.zib.de), are promising. According to practical modeling requirements, certain edges are mandatory and must belong to the spanning tree associated to the Min FCB solution. The above-mentioned instance contains 80 mandatory edges out of 87 tree branches, and most of the these 80 fixed edges have very high costs. As 
shown in Table 3 (instance 1 -fixed), this additional condition obviously leads to FCBs with substantially larger costs. Missing values in the table, marked with "-", are due to a missing implementation of the corresponding algorithm which deals with mandatory spanning tree edges. Lower bounds were obtained by solving the linear relaxation of the formulation in Section 4 .

Testing of electrical networks. The Min FCB problem also arises in the eventdriven simulation of electrical circuits [3]. The electrical network is decomposed into current sources (represented by branches in the tree) and voltage sources (represented by chords in the co-tree). Fundamental cycles are then used to compute the voltage across the current sources, and fundamental cuts to determine the current through the voltage sources. When the network evolves in time, it is necessary to perform edge swapping, and the best choice appears to be the one minimizing the length of the fundamental cycles. The instance gm19 (from A. Brambilla, DEI, Politecnico di Milano) has 414 nodes and 1091 edges. The graph was too large for the lower bound to be computed by solving the linear relaxation of the formulation in Section 4, so we reported the minimum (non-fundamental) cycle basis cost computed by Horton's algorithm $[16]$.

\begin{tabular}{|c||cc|c|cc|cc|c|}
\hline \multicolumn{1}{|||}{} & \multicolumn{2}{|c|}{ Local search } & NT $[4]$ & \multicolumn{2}{c|}{ VNS } & \multicolumn{2}{c|}{ TS } & Bound \\
\hline Instance & FCB cost & Time & FCB cost & FCB cost & Time & FCB cost & Time & FCB cost \\
\hline timtab2 & 40520 & $0.7 \mathrm{~s}$ & 50265 & $39801^{*}$ & $30 \mathrm{~s}$ & 39841 & $30 \mathrm{~s}$ & 31220.534 \\
1-fixed & 46072 & $0.13 \mathrm{~s}$ & - & - & - & $46002^{*}$ & $30 \mathrm{~s}$ & 39907.96 \\
\hline gm19 & 2592 & $1.5 \mathrm{~s}$ & 2662 & $2584^{*}$ & $600 \mathrm{~s}$ & $2584^{*}$ & $600 \mathrm{~s}$ & $2267^{\dagger}$ \\
\hline
\end{tabular}

Table 3

Computational results for real-life applications. Best values are marked with * CPU times for the NT heuristic are approximately 0s. The bound marked with ${ }^{\dagger}$ is given the cost of the MCB computed by Horton's algorithm.

\subsection{TUM cycle basis test set}

A collection of 2D and 3D toroidal graphs and of hypercubic graphs are available from http://www-m9.ma.tum.de/dm/cycles/mhuber. This website at Technische Universität München (TUM) also contains the costs of the minimum (not necessarily fundamental) cycle bases, which provide lower bounds to the minimum FCB costs. Toroidal graphs are $D$-dimensional grids of $n^{D}$ nodes each of which is adjacent to each neighboring node, with wrap-around adjacency at the border. Computational results (for a subset of the instances) are reported in Tables 4 and 5. Lower bounds, reported in the last column, refer to the minimal non-fundamental cycle bases.

For hypercubic graphs, the locally optimal FCB found by the initial constructive heuristic was never improved by edge-swapping heuristics. This is strong 
Table 4

\begin{tabular}{|c||cc|cc|c|c|c|}
\hline \multicolumn{1}{|c||}{} & \multicolumn{2}{c|}{ LS } & \multicolumn{2}{c|}{ NT [4] } & VNS & TS & Bound \\
\hline$n$ & Cost & Time & Cost & Time & Cost & Cost & Cost \\
\hline 5 & 140 & 0 & 154 & 0 & $138^{*}$ & $138^{*}$ & 106 \\
10 & 770 & 0.38 & 834 & 0 & $738^{*}$ & $738^{*}$ & 416 \\
15 & 2004 & 5.86 & 2372 & 0.01 & 1930 & $1926^{*}$ & 926 \\
20 & 3884 & 21.05 & 4856 & 0.03 & $3822^{*}$ & 3868 & 1636 \\
25 & 6650 & 80.46 & 8792 & 0.06 & $6414^{*}$ & 6650 & 2546 \\
30 & 9680 & 241.73 & 14076 & 0.09 & 9674 & $9666^{*}$ & 3656 \\
33 & 11988 & 442.8 & 18296 & 0.14 & $11976^{*}$ & 11994 & 4418 \\
\hline
\end{tabular}

Computational results for some 2D toroidal graphs from the TUM test set. Values marked with * denote the best value found for the instance.

computational evidence that the initial constructive heuristic actually finds the global optimum.

Table 5

\begin{tabular}{|c||cc|cc|c|c|c|}
\hline \multicolumn{1}{|c||}{} & \multicolumn{2}{c|}{ LS } & \multicolumn{2}{c|}{ NT [4] } & VNS & TS & Bound \\
\hline$n$ & Cost & Time & Cost & Time & Cost & Cost & Cost \\
\hline 5 & 1609 & 2.25 & 1771 & 0.02 & $1567^{*}$ & $1567^{*}$ & 1007 \\
6 & 3062 & 16.93 & 3242 & 0.04 & $3024^{*}$ & 3028 & 1738 \\
7 & 5409 & 58.87 & 5895 & 0.09 & 5409 & $5361^{*}$ & 2757 \\
8 & 8234 & 107.33 & 9030 & 0.14 & $8210^{*}$ & 8214 & 4112 \\
9 & $13105^{*}$ & 662.11 & 14525 & 0.61 & 13105 & 13105 & 5851 \\
10 & $17890^{*}$ & 1037.36 & 20248 & 1.33 & 17890 & 17890 & 8022 \\
\hline
\end{tabular}

Computational results for the 3D toroidal graphs from the TUM test set. Values marked with * denote the best value found for the instance.

\section{Concluding remarks}

We described and investigated new heuristics, based on edge swaps, for tackling the Min FCB problem. Compared to existing tree-growing procedures, our local search algorithm and simple implementation of the VNS and TS metaheuristics look very promising, even though computationally more intensive. We established structural results that allow an efficient implementation of the proposed edge swaps. We presented a new MIP formulation whose linear relaxation provides tighter lower bounds than known formulations on several classes of graphs. Finally, we tested LS, NT, VNS and TS heuristics on several classes of graphs. Our VNS and TS implementations are on average roughly equivalent in performance, and they compared favorably with straight local search but have higher computational requirements. 


\section{Acknowledgements}

We are grateful to two anonymous referees for numerous comments that greatly improved the paper.

\section{References}

[1] E. Amaldi, L. Liberti, N. Maculan, F. Maffioli, Efficient edge-swapping heuristics for finding minimum fundamental cycle bases, in: C. Ribeiro, S. Martins (Eds.), Experimental and Efficient Algorithms - WEA2004 Proceedings, Vol. 3059 of LNCS, Springer-Verlag, 2004, pp. 15-29.

[2] G. Kirchhoff, Über die aufösung der gleichungen, auf welche man bei der untersuchungen der linearen verteilung galvanischer ströme geführt wird, Poggendorf Annalen Physik 72 (1847) 497-508.

[3] A. Brambilla, A. Premoli, Rigorous event-driven (red) analysis of largescale nonlinear rc circuits, IEEE Transactions on Circuits and Systems-I: Fundamental Theory and Applications 48 (8) (2001) 938-946.

[4] N. Deo, N. Kumar, J. Parsons, Minimum-length fundamental-cycle set problem: New heuristics and an empirical investigation, Congressus Numerantium 107 (1995) 141-154.

[5] C. Liebchen, R. Möhring, A case study in periodic timetabling, in: D. Wagner (Ed.), Electronic Notes in Theoretical Computer Science, Vol. 66, Elsevier, 2002.

[6] C. Liebchen, Finding short integral cycle bases for cyclic timetabling, in: Algorithms - ESA2003 Proceedings, Vol. 2832 of LNCS, Springer-Verlag, 2003, pp. $715-726$.

[7] E. J. Sussenouth, A graph theoretical algorithm for matching chemical structures, J. Chem. Doc. 5 (1965) 36-43.

[8] P. Vismara, Reconnaissance et représentation d'éléments structuraux pour la description d'objets complexes. application à l'élaboration de stratégies de synthèse en chimie organique, Ph.D. thesis, Université de Montpellier II, France (1995).

[9] E. Hubicka, M. Sysło, Minimal bases of cycles of a graph, in: Recent Advances in Graph Theory, 2nd Czech Symposium in Graph Theory, Academia, Prague, 1975, pp. 283-293.

[10] M. Sysło, An efficient cycle vector space algorithm for listing all cycles of a planar graph, Colloquia Mathematica Societatis János Bolyai (1978) 749-762.

[11] M. Sysło, On cycle bases of a graph, Networks 9 (1979) 123-132. 
[12] M. Sysło, On some problems related to fundamental cycle sets of a graph, in: R. Chartrand (Ed.), Theory of Applications of Graphs, Wiley, New York, 1981, pp. $577-588$.

[13] M. Sysło, On some problems related to fundamental cycle sets of a graph: Research notes, Discrete Mathematics (Banach Centre Publications, Warsaw) 7 (1982) 145-157.

[14] M. Sysło, On the fundamental cycle set graph, IEEE Transactions on Circuits and Systems 29 (3) (1982) 136-138.

[15] D. Hartvigsen, E. Zemel, Is every cycle basis fundamental?, Journal of Graph Theory 13 (1) (1989) 117-137.

[16] J. Horton, A polynomial-time algorithm to find the shortest cycle basis of a graph, SIAM Journal of Computing 16 (2) (1987) 358-366.

[17] T. Kavitha, K. Mehlhorn, D. Michail, K. Paluch, A faster algorithm for minimum cycle bases of graphs, in: Proceedings of ICALP, Vol. 3124 of LNCS, Springer-Verlag, 2004, pp. 846-857.

[18] E. Amaldi, R. Rizzi, Personal communication .

[19] N. Deo, G. Prabhu, M. Krishnamoorthy, Algorithms for generating fundamental cycles in a graph, ACM Transactions on Mathematical Software 8 (1) (1982) $26-42$.

[20] G. Galbiati, R. Rizzi, E. Amaldi, On the approximability of the minimum strictly fundamental cycle bases problem, Technical Report, DEI, Politecnico di Milano, 2007.

[21] K. Paton, An algorithm for finding a fundamental set of cycles of a graph, Communications of the ACM 12 (9) (1969) 514-518.

[22] B. Y. Wu, G. Lancia, V. Bafna, R. Chao, K.-M. Ravi, C. Tang, A polynomialtime approximation scheme for minimum routing cost spanning trees, SIAM Journal on Computing 29 (1999) 761-778.

[23] M. Fischetti, G. Lancia, P. Serafini, Exact algorithms for minimum routing cost trees, Networks 93 (3) (2002) 161-173.

[24] H. Gabow, E. Myers, Finding all spanning trees of directed and undirected graphs, SIAM Journal of Computing 7 (3) (1978) 280-287.

[25] A. Shioura, A. Tamura, T. Uno, An optimal algorithm for scanning all spanning trees of undirected graphs, SIAM Journal of Computing 26 (3) (1997) 678-692.

[26] M. Elkin, Y. Emek, D. Spielman, S.-H. Teng, Lower-stretch spanning trees, in: STOC '05: Proceedings of the 37th Annual ACM Symposium on Theory of computing, ACM, New York, 2005, pp. 494-503.

[27] M. Elkin, C. Liebchen, R. Rizzi, New length bounds for cycle bases, Information Processing Letters 104 (2007) 186-193. 
[28] K. Mehlhorn, S. Näher, Leda, a platform for combinatorial and geometric computing, Communications of the ACM 38 (1) (1995) 96-102.

[29] P. Hansen, N. Mladenović, Variable neighbourhood search, in: P. Pardalos, M. Resende (Eds.), Handbook of Applied Optimization, Oxford University Press, Oxford, 2002.

[30] A. Hertz, E. Taillard, D. de Werra, Tabu search, in: E. Aarts, J. Lenstra (Eds.), Local Search in Combinatorial Optimization, Wiley, Chichester, 1997, pp. 121136.

[31] P. Hansen, N. Mladenović, Variable neighbourhood search: Principles and applications, European Journal of Operations Research 130 (2001) 449-467.

[32] L. Liberti, E. Amaldi, N. Maculan, F. Maffioli, Mathematical models and a constructive heuristic for finding minimum fundamental cycle bases, Yugoslav Journal of Operations Research 15 (1) (2005) 15-24.

[33] ILOG, ILOG CPLEX 8.0 User's Manual, ILOG S.A., Gentilly, France (2002).

[34] B. Guta, Subgradient optimization methods in integer programming, with an application to a radiation therapy problem, Ph.D. thesis, Kaiserslautern University, Germany (2003).

[35] L. Lissoni, Implementazione di un algoritmo efficiente per determinare una base di cicli minima di un grafo, tesi di Laurea, DEI, Politecnico di Milano, Italy (2003).

[36] C. Liebchen, G. Wünsch, E. Köhler, A. Reich, R. Rizzi, Benchmarks for strictly fundamental cycle bases, in: C. Demetrescu (Ed.), Experimental Algorithms WEA 2007, Vol. 4525 of LNCS, Springer, New York, 2007, pp. 365-378.

[37] P. Serafini, W. Ukovich, A mathematical model for periodic scheduling problems, SIAM Journal of Discrete Mathematics 2 (4) (1989) 550-581.

[38] N. Alon, R. Karp, D. Peleg, D. West, A graph-theoretic game and its application to the $k$-server problem, SIAM Journal on Computing (1995).

\section{Appendix A: proof of Theorem 9}

To establish that for each $h \in T$ such that $h \neq e$ and $f \in \delta_{T}^{h}$ we have $\pi\left(\delta_{T}^{h}\right)=\delta_{T}^{h} \triangle \delta_{T}^{e}$, we distinguish four separate cases, illustrated in Figures 912 .

We prove that: (1) $g \in \delta_{T}^{h} \cap \delta_{T}^{e} \Rightarrow g \notin \pi\left(\delta_{T}^{h}\right),(2) g \notin \delta_{T}^{h} \cup \delta_{T}^{e} \Rightarrow g \notin \pi\left(\delta_{T}^{h}\right)$,

(3) $g \in \delta_{T}^{h} \backslash \delta^{e} \Rightarrow g \in \pi\left(\delta_{T}^{h}\right)$, and (4) $g \in \delta^{e} \backslash \delta_{T}^{h} \Rightarrow g \in \pi\left(\delta_{T}^{h}\right)$.

When there is no ambiguity, $\delta_{T}^{e}$ is written $\delta^{e}$. 
Claim 1: $g \in \delta^{h} \cap \delta^{e} \Rightarrow g \notin \pi\left(\delta^{h}\right)$.

Proof. Since $g \in \delta^{h}$ there are shortest paths $p_{1}^{T}, p_{2}^{T}$ connecting $g, h$ and not
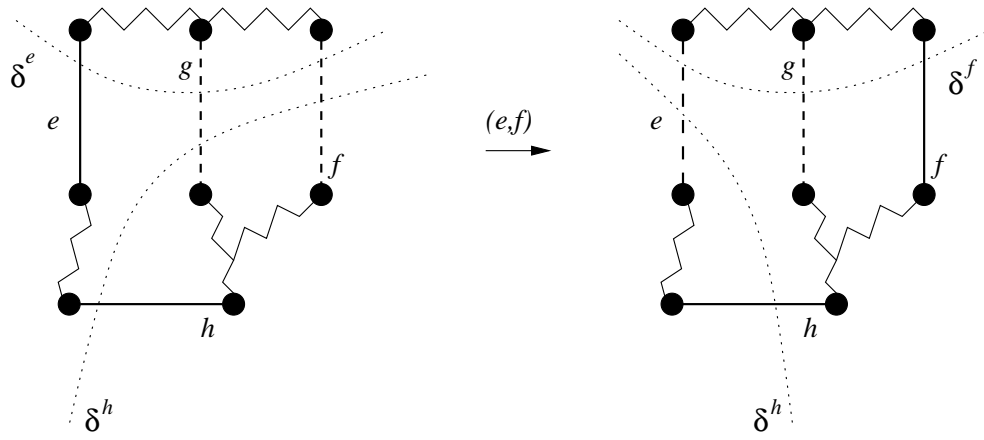

Fig. 9. Claim 1: If $g \in \delta^{h} \cap \delta^{e}$ then $g \notin \pi\left(\delta^{h}\right)$.

containing $h$, such that $p_{1}^{T} \subseteq S_{T}^{h}$ and $p_{2}^{T} \subseteq \bar{S}_{T}^{h}$. Since $g \in \delta^{e}$, either $p_{1}^{T}$ or $p_{2}^{T}$ must contain $e$, but not both. Assume w.l.o.g. that $e \in p_{1}, e \notin p_{2}$ (i.e., $e \in S_{T}^{h}$ ). Thus $\pi$ sends $p_{1}^{T}$ to a path $p_{1}^{\pi T}$ containing $f$, whereas $p_{2}^{\pi T}=p_{2}^{T}$. Thus $P_{\pi T}^{*}(h, g)=\left\{p_{1}^{\pi T}, p_{2}^{\pi T}\right\}$. Since $f \in \delta_{T}^{h}$, there exist shortest paths $q_{1}^{T} \subseteq S_{T}^{h}$ and $q_{2}^{T} \subseteq \bar{S}_{T}^{h}$ connecting $f, h$ and not containing $h$. Because $q_{2}^{T} \subseteq \bar{S}_{T}^{h}, e \notin q_{2}^{T}$. In $\pi T, q_{2}^{T}$ can be extended to a path $q^{\pi T}=q_{2}^{T} \cup\{f\}$. By Proposition $7 g \in \delta_{\pi T}^{f}$. Thus in $\pi T$ there exist paths $r_{1}^{\pi T}$ in $S_{\pi T}^{f}=S_{T}^{e}$ and $r_{2}^{\pi T}$ in $\bar{S}_{\pi T}^{f}=\bar{S}_{T}^{e}$ connecting $f, g$ and not containing $f$. Notice that the path $q^{\pi T} \cup r_{1}^{\pi T}$ connects the endpoint of $h$ in $\bar{S}_{T}^{h}$ and $g$, and $p_{2}^{\pi T}$ connects the same endpoint of $h$ with the opposite endpoint of $g$. Thus $p_{1}^{\pi T}=\{h\} \cup q^{\pi T} \cup r_{1}^{\pi T}$, which means that $h \in p_{1}^{\pi T}$, i.e., $P_{\pi T}^{*}(h, g) \neq \bar{P}_{\pi T}(h, g)$. By Lemma 6 , the claim is proved.

Claim 2: $g \notin \delta^{h} \cup \delta^{e} \Rightarrow g \notin \pi\left(\delta^{h}\right)$.

Proof. By hypothesis, $g \in S_{T}^{e} \cap S_{T}^{h}$ or $g \in \bar{S}_{T}^{e} \cap \bar{S}_{T}^{h}$. Assume the former w.l.o.g.
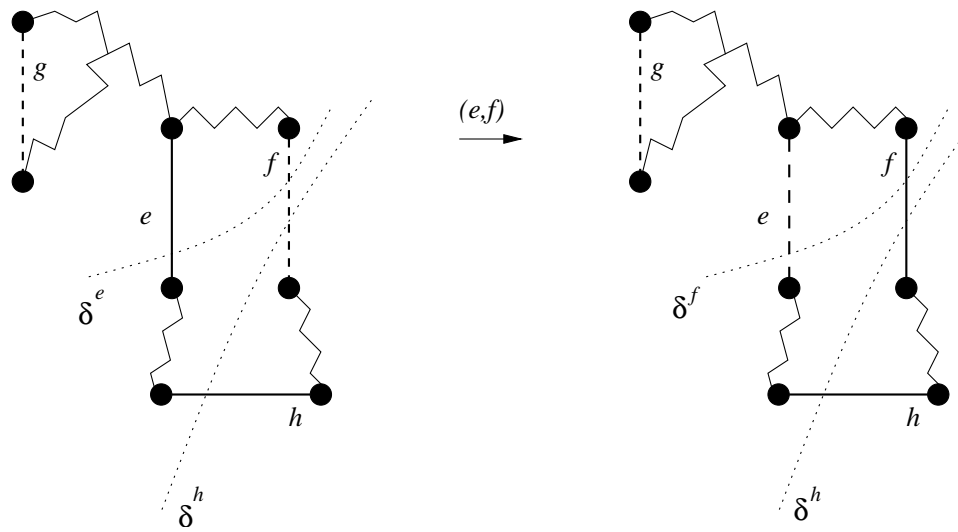

Fig. 10. Claim 2: If $g \notin \delta^{h} \cup \delta^{e}$ then $g \notin \pi\left(\delta^{h}\right)$.

Let $p_{1}^{T}, p_{2}^{T}$ be unique shortest paths from $h$ to $g$, and assume $h \in p_{1}^{T}$. Thus $p_{2}^{T} \subseteq S_{T}^{h}$. Since $g \notin \delta^{e}$, there are unique shortest paths $q_{1}^{T}, q_{2}^{T}$ from $e$ to $g$ such that $e$ is in one of them, assume $e \in q_{1}^{T}$. Since both $h, e \in T$, there is a 
shortest path $r^{T}$ between one of the endpoints of $h$ and one of the endpoints of $e$, while the opposite endpoints are linked by the path $\{h\} \cup r^{T} \cup\{e\}$. Suppose $e \in p_{1}^{T}$. Then since both endpoints of $g$ are reachable from $e$ via $q_{1}^{T}, q_{2}^{T}$, and $e$ is reachable from $h$ through $r^{T}$, it means that $e \in p_{2}^{T}$. Conversely, if $e \notin p_{1}^{T}$, then $e \notin p_{2}^{T}$. Thus, we consider two cases. If $e$ is not in the paths from $h$ to $g$, then $\pi$ fixes those paths, i.e., $h \in p_{1}^{\pi T}$ and $h \notin p_{2}^{\pi T}$, that is $g \notin \delta^{h}$. If $e$ is in the paths from $h$ to $g$, then both the unique shortest paths $p_{1}^{\pi T}$ and $p_{2}^{\pi T}$ connecting $h$ and $g$ in $\pi T$ contain $f$. Since $g \notin \delta_{\pi T}^{f}=\delta_{T}^{e}$, there are shortest paths $s_{1}^{\pi T}, s_{2}^{\pi T}$ connecting $f$ to $g$ one of which, say $s_{1}^{\pi T}$, contains $f$. Moreover, since both $h, f \in T$, there is a shortest path $u^{\pi T}$ connecting one of the endpoints of $h$ to one of the endpoints of $f$, the other shortest path between the opposite endpoints being $\{h\} \cup u^{\pi T} \cup\{f\}$. Thus, either $p_{1}^{\pi T}=u^{\pi T} \cup s_{1}^{\pi T}$ and $p_{2}^{\pi T}=\{h\} \cup u^{\pi T} \cup\{f\} \cup s_{2}^{\pi T}$, or $p_{1}^{\pi T}=u^{\pi T} \cup\{f\} \cup s_{2}^{\pi T}$ and $p_{2}^{\pi T}=\{h\} \cup u^{\pi T} \cup s_{1}^{\pi T}$. Either way, one of the paths contains $h$. By Lemma 6, the claim is proved.

Claim 3: $g \in \delta^{h} \backslash \delta^{e} \Rightarrow g \in \pi\left(\delta^{h}\right)$.

Proof. Since $g \in \delta^{h}$, there are shortest paths $p_{1}^{T} \subseteq S_{T}^{h}, p_{2}^{T} \subseteq \bar{S}_{T}^{h}$ connecting $h$
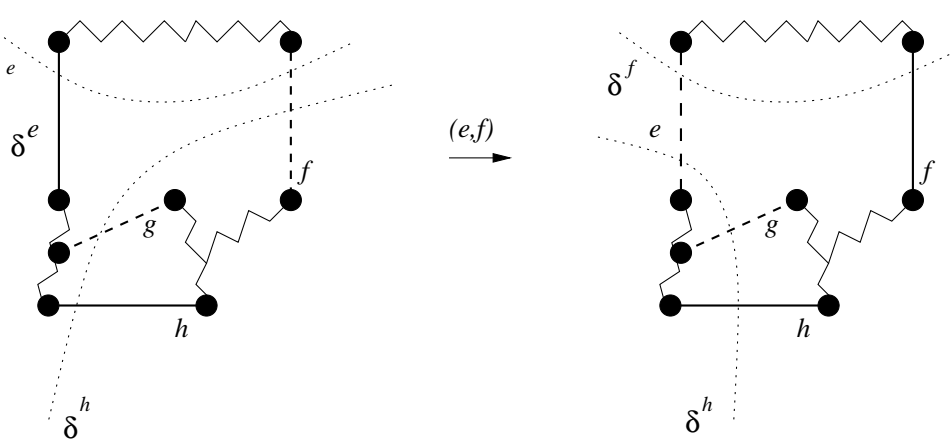

Fig. 11. Claim 3: If $g \in \delta^{h}$ and $g \notin \delta^{e}$, then $g \in \pi\left(\delta^{h}\right)$.

and $g$, none of which contains $h$. Assume w.l.o.g. $e \in S_{T}^{h}$. Suppose $e \in p_{1}^{T}$, say $p_{1}^{T}=q^{T} \cup\{e\} \cup r^{T}$. Consider $s^{T}=q^{T} \cup\{h\} \cup p_{2}^{T}$ and $r^{T}$. These are a pair of shortest paths connecting $e$ and $g$ such that $e$ does not belong to either; i.e., $g \in \delta_{T}^{e}$, which contradicts the hypothesis. Thus $e \notin p_{1}^{T}$, i.e., $\pi$ fixes paths $\pi_{1}^{T}, \pi_{2}^{T}$; thus $\bar{P}_{\pi T}(h, g)=\bar{P}_{T}(h, g)=P_{T}^{*}(h, g)=P_{\pi T}^{*}(h, g)$, which proves the claim.

Claim 4: $g \in \delta^{e} \backslash \delta^{h} \Rightarrow g \in \pi\left(\delta^{h}\right)$.

Proof. First consider the case where $e, g \in S_{T}^{h}$. Since $g \notin \delta_{T}^{h}$, the shortest paths $p_{1}^{T}, p_{2}^{T}$ connecting $h, g$ are such that one of them contains $h$, say $h \in p_{1}^{T}$, whilst $p_{2}^{T} \subseteq S_{T}^{h}$. Since $g \in \delta^{e}$, there are shortest paths $q_{1}^{T}, q_{2}^{T}$ entirely in $S_{T}^{h}$, connecting $e, g$ such that neither contains $e$. Since both $e, h \in T$ there is a shortest path $r^{T} \subseteq S_{T}^{h}$ connecting an endpoint of $h$ to an endpoint of $e$, the opposite endpoints being joined by $\{h\} \cup r^{T} \cup\{e\}$. Thus w.l.o.g. $p_{1}^{T}=\{h\} \cup r^{T} \cup\{e\} \cup q_{1}^{T}$. 

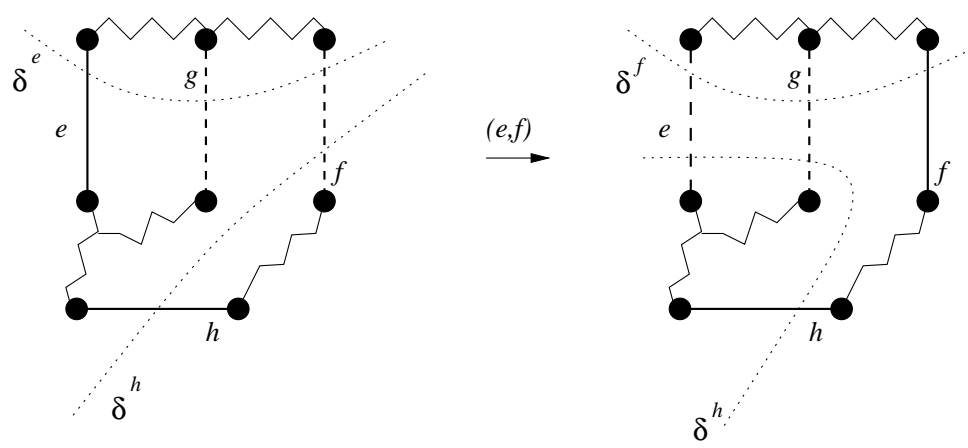

Fig. 12. Claim 4: If $g \notin \delta^{h}$ and $g \in \delta^{e}$, then $g \in \pi\left(\delta^{h}\right)$.

Since the path $r^{T} \cup q_{2}^{T}$ does not include $e$ and connects $h, g$, $e \notin p_{2}^{T}$. Thus $p_{2}^{\pi T}=p_{2}^{T}$ and $h \notin p_{2}^{\pi T}$. On the other hand $\pi$ sends $p_{1}^{T}$ to a unique shortest path $p_{1}^{\pi T}$ connecting $h, g$ that includes $f$. Since $f \in \delta_{T}^{h}$, there are shortest paths $s_{1}^{T} \subseteq S_{T}^{h}, s_{2}^{T} \subseteq \bar{S}_{T}^{h}$ that do not include $h$. Since $p_{2}^{T} \subseteq S_{T}^{h}, s_{1}^{T}$ may only touch the same endpoint of $h$ as $p_{2}^{T}$. Thus the endpoint of $h$ touched by $p_{1}^{T}$ also originates $s_{2}^{T}$. Since $s_{2}^{T} \subseteq \bar{S}_{T}^{h}, h \notin s_{2}^{T}$. Since $p_{1}^{\pi T}$ joins $h, g$, contains $f$ and is shortest, $p_{1}^{\pi T}=s_{1}^{T} \cup\{f\} \cup u$, where $u^{\pi T}$ is a shortest path from $f$ to $g$ (which exists because by Proposition $7 g \in \delta_{\pi T}^{f}$ ), which shows that $h \notin p_{1}^{\pi T}$. Thus by Lemma $6, g \in \pi\left(\delta^{h}\right)$. The second possible case is that $e \in S_{T}^{h}, g \in \bar{S}_{T}^{h}$. Since $g \in \delta_{T}^{e}$ there are shortest paths $p_{1}^{T}, p_{2}^{T}$ connecting $e, g$ such that neither includes $e$. Assume w.l.o.g. $h \in S_{T}^{e}$. Since $e, g$ are partitioned by $\delta_{T}^{h}$, exactly one of $p_{1}^{T}, p_{2}^{T}$ includes $h$ (say $h \in p_{1}^{T}$, which implies $p_{1}^{T} \subseteq S_{T}^{h}$ ). Let $q_{1}^{T}$ be the sub-path of $p_{1}^{T}$ joining $h$ and $g$ and not including $h$, and let $r^{T}$ be the sub-path of $p_{1}^{T}$ joining $h$ and $e$ and not including $h$. Let $q_{2}^{T}=r^{T} \cup\{e\} \cup p_{2}^{T}$. We have that $q_{2}$ is a shortest path joining $h, g$ not including $h$. Thus $\bar{P}_{T}(h, g)=\left\{q_{1}^{T}, q_{2}^{T}\right\}=P_{T}^{*}(h, g)$, and by Lemma $6 g \in \delta_{T}^{h}$, which is a contradiction.

\section{Appendix B: Comparison of best swap algorithms}

Computational comparison of the local search algorithm using LCA-based and symmetric difference-based implementations. Average of user CPU times ratio LCA-based/Symmdiff-based: 21.381733, standard deviation: 10.145212. 


\begin{tabular}{|c|c|c|c|}
\hline Instance name & FCB Cost & LCA-based (seconds) & Symmdiff-based (seconds) \\
\hline euclid-40_0.8 & 13698.1 & 261.345 & 12.4031 \\
\hline euclid-50_0.3 & 10085.6 & 66.1819 & 3.69544 \\
\hline euclid-75_0.2 & 16878.5 & 284.533 & 10.2924 \\
\hline hypercube-7_0 & 603.327 & 146.554 & 17.8823 \\
\hline hypercube-7_-1 & 512.612 & 135.625 & 12.5261 \\
\hline hypercube-7_- 2 & 573.665 & 128.384 & 10.0195 \\
\hline hypercube-7_-3 & 515.37 & 194.44 & 20.1819 \\
\hline hypercube-7_4 & 583.176 & 174.868 & 14.1029 \\
\hline hypercube-7_5 & 583.601 & 163.057 & 18.6732 \\
\hline hypercube- $-7-6$ & 568.314 & 150.146 & 16.8114 \\
\hline hypercube-7-7 & 577.642 & 149.693 & 15.0577 \\
\hline hypercube-7_8 & 555.623 & 152.57 & 18.2222 \\
\hline hypercube-7_-9 & 553.632 & 140.768 & 15.2127 \\
\hline hypercube-7 & 1986 & 1.75073 & 8.75267 \\
\hline mesh-15_0 & 539.587 & 53.8818 & 1.57976 \\
\hline mesh-15_1 & 533.461 & 58.3101 & 1.82272 \\
\hline mesh-15_2 & 513.962 & 53.0169 & 1.71174 \\
\hline mesh-15_3 & 491.852 & 53.0849 & 2.05369 \\
\hline mesh-15_4 & 536.154 & 59.5309 & 2.34864 \\
\hline mesh-15_5 & 496.06 & 56.0165 & 2.22666 \\
\hline mesh-15_6 & 509.269 & 41.6437 & 1.67375 \\
\hline mesh-15_7 & 461.934 & 48.8766 & 1.3318 \\
\hline mesh-15_8 & 530.033 & 46.3799 & 1.46478 \\
\hline mesh-15_9 & 551.304 & 46.4889 & 1.74074 \\
\hline mesh -15 & 1318 & 22.7785 & 0.955855 \\
\hline random-40_0.8 & 1846 & 3.54346 & 0.086986 \\
\hline random-50_0.3_0 & 279.792 & 91.1901 & 9.70252 \\
\hline random-50_0.3_1 & 323.47 & 101.088 & 7.18991 \\
\hline random-50_0.3_2 & 318.589 & 90.3893 & 10.5884 \\
\hline random-50_0.3_3 & 307.708 & 86.9288 & 6.72598 \\
\hline random-50_0.3_4 & 341.978 & 73.8478 & 5.75413 \\
\hline random-50_0.3-5 & 351.295 & 117.428 & 10.4234 \\
\hline random-50_0.3_6 & 338.125 & 91.2041 & 4.05738 \\
\hline random-50_0.3-7 & 327.712 & 138.806 & 10.0675 \\
\hline random-50_0.3_8 & 312.191 & 98.1721 & 4.83727 \\
\hline random-50_0.3_9 & 347.057 & 146.437 & 10.0145 \\
\hline random-50_0.3 & 1410 & 11.9282 & 0.652901 \\
\hline random-75_0.2 & 2239 & 82.7704 & 2.36664 \\
\hline torus-15_0 & 779.19 & 136.352 & 12.3541 \\
\hline torus -15 & 684.073 & 102.291 & 12.927 \\
\hline torus $-15 \_2$ & 694.008 & 79.7819 & 9.8385 \\
\hline torus-15_3 & 688.822 & 98.3271 & 11.7462 \\
\hline torus -15.4 & 679.94 & 87.3487 & 13.6699 \\
\hline torus $-15-5$ & 677.581 & $\begin{array}{l}0 \\
77.7322\end{array}$ & 12.3981 \\
\hline torus $-15 \_6$ & 729.846 & 97.2192 & 12.0492 \\
\hline torus $-15 \_7$ & 693.137 & 95.9574 & 13.191 \\
\hline torus-15_8 & 785.313 & 103.293 & 12.7601 \\
\hline torus $-15 \_9$ & 761.546 & 84.1552 & 10.5694 \\
\hline torus -15 & 1994 & 46.8199 & 6.11907 \\
\hline triangle-20_0 & 630.693 & 312.561 & 7.50186 \\
\hline triangle-20_1 & 676.275 & 297.625 & 6.92595 \\
\hline triangle-20_2 & 644.77 & 264.222 & 6.30804 \\
\hline triangle-20_3 & 637.528 & 296.559 & 6.33004 \\
\hline triangle-20_4 & 655.714 & 250.214 & 4.96824 \\
\hline triangle-20_5 & 623.176 & 273.085 & 5.95609 \\
\hline triangle-20_6 & 633.518 & $\begin{array}{l}204.851 \\
3040\end{array}$ & 5.9101 \\
\hline triangle-20_7 & 553.599 & 235.029 & 4.20336 \\
\hline triangle-20_8 & 617.789 & 259.001 & 5.3002 \\
\hline triangle-20_9 & 655.251 & 286.719 & 7.02793 \\
\hline triangle -20 & 1934 & 115.115 & 2.98455 \\
\hline
\end{tabular}

